\title{
Temporal dynamics of the hypnotic response
}

Hernán Anlló 1,5, Joshua Hagège1,6 \& Jérôme Sackur 2,3,4

1. Watanabe Laboratory. School of Fundamental Science and Engineering, Waseda University, Tokyo, Japan.

2. Laboratoire de Sciences Cognitives et Psycholinguistique, Département d'Études Cognitives, École Normale Supérieure, PSL Research University, École des Hautes Études en Sciences Sociales, Centre National de la Recherche Scientifique, Paris, France

3. École des Hautes Études en Sciences Sociales, Paris, France.

4. École Polytechnique, Palaiseau, France

5. Hypnosis Research Team, Centre Hospitalier de Bligny, Briis-sous-Forges, France.

6. Sorbonne Université, Médecine. Paris, France.

jerome.sackur@gmail.com

joshua.hageg@gmail.com

hernan.anllo@cri-paris.org (Corresponding Author) - Watanabe Cognitive

Science Laboratory School of Fundamental Science and Engineering. Waseda

University. Rm 407-1B, 59th BIdg. 3-4-1 Ohkubo, Shinjuku, Tokyo 169-8555, Japan 


\section{Highlights}

* Our hypnotic procedure modulated the size of the Emotional Attentional Blink

${ }^{*}$ The impact of hypnosis was limited to late targets leading to response preparation

* We isolated the roles of task-set updating and response preparation in hypnotic responding

\section{Keywords}

Hypnosis; Attentional Blink; Anger Superiority; Emotional Numbing; Attentional Task Set; Cognitive Control

\section{ABSTRACT}

To establish the temporal dynamics of hypnotic responding, we composed an Attentional Blink paradigm in which both the first and second targets were faces, either expressing neutral or angry emotions. We then suppressed the emotional advantage of angry faces by means of a "hypnotic numbing" suggestion. Hypnosis attenuated only the emotional salience of the second target (T2), suggesting that hypnosis' effects were time and resource dependent. Additionally, we found that hypnosis fostered changes in the attentional task-set of all participants, regardless of their hypnotizability. By implementing drift-diffusion decision modelling, we found that hypnosis lowered decision thresholds. These results portray hypnotic responding as a two-tiered process: first, hypnosis alters the attentional task-set, then, provided processing and response preparation are not interrupted, a hypnotic response based on this altered task-set is produced through late cognitive control strategies dependent on hypnotic susceptibility. 


\section{STATEMENT OF RELEVANCE}

Over the past two decades hypnosis research has continuously improved; with a better understanding of its mechanisms, came the possibility of using it as a tool for tackling important general cognitive issues (Terhune et al., 2017). Here, we outline the temporal dynamics of hypnosis, and its link to attention and cognitive control. We also show that, to some extent, hypnosis can influence the attentional task-sets of all individuals regardless of their hypnotic susceptibility. Such findings should be of interest to a broad audience of clinical and cognitive researchers, including those working on the mechanisms behind social influence, cognitive control, attentional task-sets and emotion processing. A better understanding of hypnosis constitutes an inescapable step in achieving a well-rounded "psychology of influence". Moreover, hypnosis and its so-often-romanticized mechanisms awaken an enormous interest in the general public: this tasks us with the responsibility of separating fact from fiction through replicable research.

\section{INTRODUCTION}

Hypnosis has been subjected to renewed scrutiny. We now know more about its range (i. e., what processes are amenable to hypnotic modulation; Terhune, Cleeremans, 
Raz \& Lynn, 2017), its individual traits (i. e., hypnotic susceptibility; Terhune \& Cardeña, 2014), and have new theoretical models (Martin \& Pacherie, 2019). Yet, the time course of hypnosis' deployment remains largely unknown. Namely, the steps through which hypnosis acts, and crucially, when do these steps take place throughout the perceptual process. Does hypnosis exert its effects by modifying participants' general cognitive make-up? Or is it rather a single cognitive process that deploys over time? Here, we set out to clarify this issue by using a well-tried experimental model (the emotional attentional blink, EAB; McHugo, Olatunji \& Zald, 2013) together with a well replicated hypnotic effect (hypnotic emotional numbing; Bryant \& Kapur, 2006). By utilizing the $E A B$ as a cognitive "bubble chamber", we tracked the temporal dynamics of hypnotic response.

Temporal attention manages the distribution of cognitive resources across a task timeline (Thorpe, Fize \& Marlot, 1996). One of the most researched experimental phenomena for probing temporal attention deprivation is the Attentional Blink (Raymond, Shapiro \& Arnell, 1992). AB paradigms typically intersperse two targets $(T 1, T 2)$ within a Rapid Serial Visual Presentation (RSVP) of distractor stimuli. When the time lag between T1 and T2 is short enough, the attentional resources invested in detecting and acting upon $\mathrm{T} 1$ fail to be diverted in time to $\mathrm{T} 2$. This causes an attentional "blink" that can impact negatively on the performance of T2-related tasks (Nieuwenstein, Potter \& Theeuwes, 2009). This impact can range from failure to detect and report T2 (Chun \& Potter, 1995) to a relative drop in performance for tasks involving T2 (de Jong, Koster, van Wees \& Martens, 2009; Maratos, Mogg \& Bradley, 2008). The $A B$ demonstrates that perceptual decisions depend on a funnel-like, immediate consolidation process that mediates the entry of perceptual information into working memory (Vogel, Luck \& Shapiro, 1998). 
The $A B$ can be enhanced or disrupted by target features evoking arousal and affect (Olivers \& Nieuwenhuis, 2006). Many studies have taken advantage of this phenomenon to explore the link between temporal attention and emotion perception (Stein, Zwickel, Ritter, Kitzmantel \& Schneider, 2009). AB paradigms employing angry or menacing faces as target stimuli have shown that the Anger Superiority Effect (ASE, i. e. the preferential processing of angry faces among crowds or series of other faces; Hansen \& Hansen, 1988) can enhance the blink selectively (de Jong, Koster, van Wees \& Martens, 2009). When an angry face, as opposed to a neutral face, is presented as $\mathrm{T} 1$, the additional attentional capture fostered by the ASE further impedes the allocation of attentional resources to T2 (de Jong, Koster, van Wees \& Martens, 2009; Maratos, Mogg \& Bradley, 2008). It has been suggested that the affective impact of $\mathrm{T} 1$ features on temporal attention is automatic in nature, and does not require stimuli to be attended, let alone reported (Arnell, Killman \& Fijavz, 2007; Most, Smith, Cooter, Levy \& Zald, 2007).

Arousal and negative values elicited by menacing stimuli have been shown to be sensitive to top-down hypnotic modulation. For example, "emotional numbing" hypnotic suggestions successfully change participants' affective response to negative stimuli. In one experiment, hypnotized participants were exposed to negative stimulation, and reported to feel no emotional disturbance or particular concern while displaying attenuated behavioral and EMG responses compared to controls (Bryant \& Kapur, 2006).

Since the ASE relies on affective appreciation (Öhman, 2002), we predicted that an "emotional numbing" hypnotic procedure applied to an EAB paradigm would attenuate affective-driven attentional capture, allowing us to track how the hypnotic modulations fare along the timeline of temporal attention deployment 
We composed an "angry vs. neutral" $A B$ paradigm consisting of two emotionallyrelevant Target faces (T1, T2), interspersed amongst a Rapid Serial Visual Presentation stream (RSVP) of distractor composite faces. All stimuli were tilted with respect to the vertical. We instructed participants to indicate whether T2 was tilted clockwise or counter-clockwise. We predicted that this paradigm would elicit an $A B$ which would be selectively enhanced or dampened depending on whether ASE favored T1 or T2 processing. Then, we embedded this paradigm within a betweengroup hypnotic comparison. Participants in the Hypnosis group underwent a hypnotic procedure aimed at reducing affective attentional modulations, while participants in the No Hypnosis group did not. Our prediction was that by hypnotically suggesting distance and emotional detachment from the angry faces' affective traits, we would successfully hamper the modulations that the ASE was expected to exert over the blink. Within both the hypnosis and no-hypnosis groups, we composed sub-groups of high, medium and low hypnotizable participants, anticipating the efficacy of hypnosis would depend on this trait. We expected the $A B$ to operate as a "temporal prism": by tracking the effects of our hypnotic procedure across the different stages of $A B$, we evaluated the temporal attention dynamics of hypnosis, from stimulus onset to response.

\section{MATERIALS \& PROCEDURES}

\subsection{Stimuli}


The experiment consisted of an "angry vs neutral" face-based Attentional Blink ( $A B)$ paradigm that both selectively enhanced (for angry T1) and hampered (for angry T2) the blink's effects, by capitalizing on the Anger Superiority effect (ASE). Each trial presented a RSVP built of three types of stimuli: neutral and angry faces (targets), and distractor faces that were blurry, composite neutral faces. A total of 40 angry and 40 neutral faces were produced as stimuli. See Stimuli in Appendix A, Extended Methods for a detailed account on stimuli production.

\subsection{Trials}

Each trial of the paradigm consisted of an RSVP stream of 16 stimuli displayed over a gray background $\left(25 \mathrm{~cd} / \mathrm{m}^{2}\right)$, at the center of the screen, for a duration of $117 \mathrm{~ms}$ each. Additionally, a blank gray frame of 17 ms would appear between stimuli to avoid facemerging. We designated angry and neutral faces as targets, and composite faces as distractors. All faces were tilted by $12^{\circ}$ either clockwise $(\mathrm{CW})$ or counterclockwise (CCW). In each stream of stimuli, Target 1 (T1) would appear in either the third, fourth or fifth position. We then selected a total of six lags: 1, 2, 3, 5, 8 and 10 for the presentation of T2. As control trials, we presented trials in which only T2 would appear, with its position calculated relative to a composite distractor face in the position of T1 (i.e., the composite filler face would too be either the third, fourth or fifth stimulus of the stream). The composite faces and their tilt were randomly selected. The main experiment consisted of a total of 600 trials, of which 120 were controls (only one target). Of the remaining 480 trials, 288 corresponded to lags 1, 2 and 3 ("Early" lags), 
and 192 to Lags 5, 8 and 10 ("Late" lags) ${ }^{1}$. These were counterbalanced for all possible target combinations (angry and neutral T1s and T2s) and tilts (CW, CCW) combinations. By the end of each RSVP, two symbolic successive cues were displayed to prompt for speeded responses. These cues asked the participant to perform the following two tasks in sequence, as fast as possible: 1 ) report the tilt of the last target face they had seen, and 2) report how many target faces had appeared in the stream. Participants were never asked about the actual emotion displayed by the targets or distractors, thus the emotion was task irrelevant. Figure 1 presents a graphical outline of the paradigm's trials.

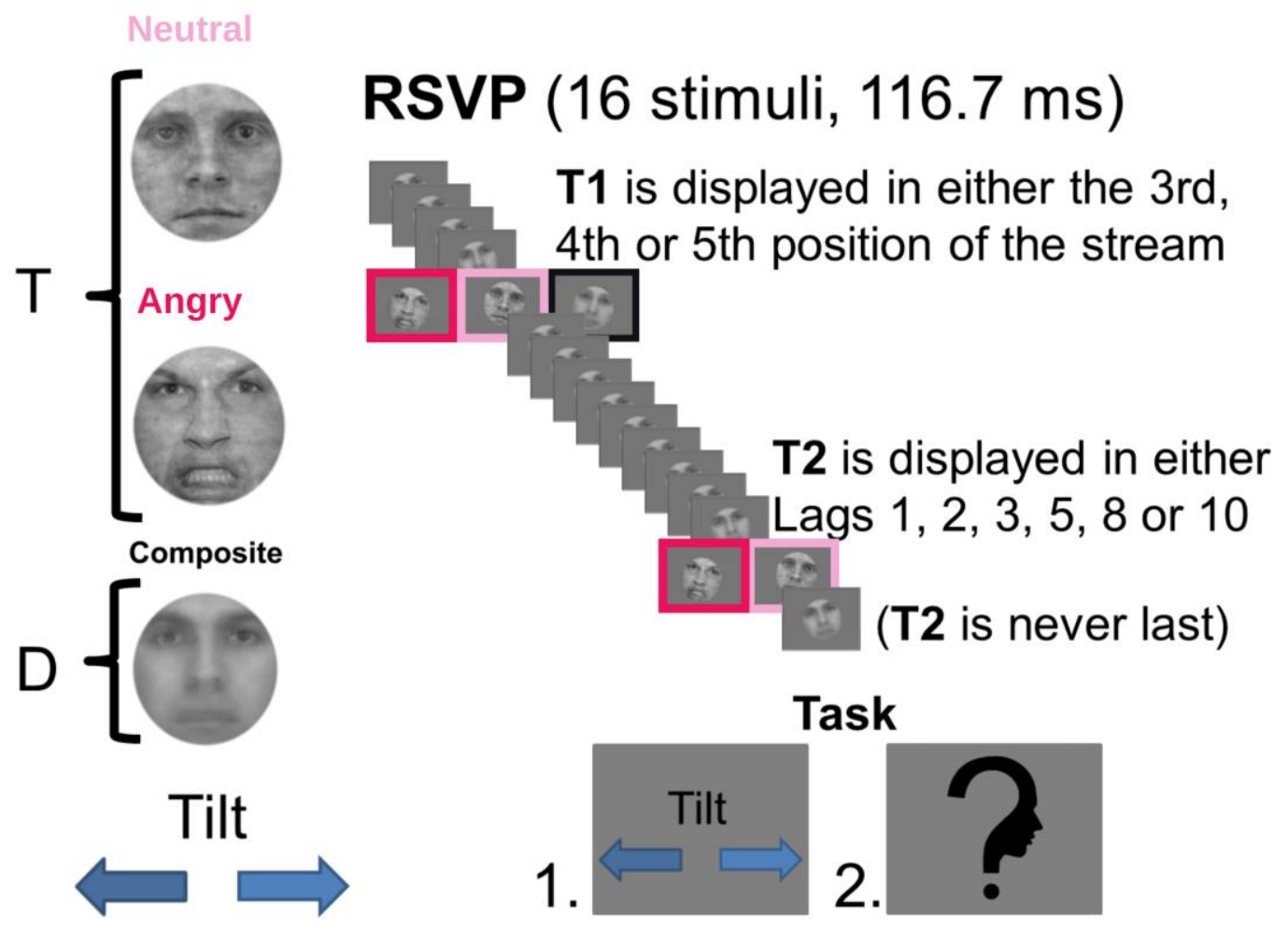

1 The difference in trial allocation was based on the literature and our pilot data, which showed performance started to plateau between lags 5 and 10. We hoped to increase the amount of trials for lags that we estimated would yield a difference in performances. 
Figure 1: Face-based Attentional Blink paradigm. The paradigm consisted of the RSVP of 16 faces, for a duration of $117 \mathrm{~ms}$ each. Angry and neutral faces were selected as targets, and composite faces as fillers. By the end of each trial, participants were presented with two subsequent response cues, and asked to perform the following two tasks in sequence, as fast as possible: 1) report the tilt of the last target face they had seen, and 2) report how many target faces had appeared in the stream. Participants were never asked about the actual emotion displayed by the targets or distractors, ensuring that their actual emotional value would remain task-irrelevant

\subsection{Participants}

Of 650 volunteers who participated in the pre-screening sessions for hypnotic susceptibility, a total of 105 right-handed, native French speakers aged between 18 and 35 (77 female) responded to the email and participated in the present experiment. Participation was voluntary, in exchange of 15 euros for a 1 hour and 30 minutes session. 49 participants were tested under hypnosis (Hypnotized Group), and 56 were tested without any sort of suggestion or hypnotic induction (Not Hypnotized Group). Interactions with all participants were scripted from first contact, so as to establish equal conditions of motivation for both groups. The Hypnosis Group was composed by 18 participants highly susceptible to hypnosis (Harvard score 9-12: Highs), 15 of medium susceptibility (score 5-8: Mediums) and 16 of low susceptibility (score 0-4: Lows). The No Hypnosis Group was composed of 14 Highs, 24 Mediums and 18 Lows. See Participants in Appendix A: Extended Methods for a detailed account of the recruitment process.

All participants signed a written consent allowing for the anonymous exploitation of the data they produced. The experiment was conducted in agreement with the Declaration 
of Helsinki (2008) and approved by the Ethics Committee of the Universite Paris Descartes (Paris 5).

\subsection{Hypnotic Induction \& suggestions}

The hypnotic induction was a variation of the Elman induction (Elman, 1984), which demands multiple rounds of eye closure, and asks the participant to "deepen" their hypnotic state with each round. The "emotional numbing" suggestion was based on Bryant's procedure (Bryant \& Kapur, 2006), suggesting distance and emotional detachment from all menacing and negative affective traits. Other secondary suggestions for safety and improved concentration were added to ensure the effect would be achieved ${ }^{2}$. Hereon, we will refer to the combination of induction and all of the suggestions as "hypnosis" and "hypnotic procedure".

By no means participants were warned that the targets they were about to see sported different emotions. Induction and suggestions were both crafted by the first author, who is a licensed clinical hypnosis practitioner. A detailed account of the induction and suggestions can be found in Appendix B of the Supplementary Methods. Participants who did not undergo the hypnosis procedure received no mention whatsoever regarding the faces' expressions, anger or menace.

\subsection{Procedure}

2 We were aware that the use of multiple suggestions, together with a formal induction, would render us unable to precise if the effect came from any particular suggestion, all of them, or even the induction itself. We decided that establishing this was beyond the scope of the present work. 
Participants were greeted by the first author, who was also responsible for the hypnosis procedure. After signing the written consent, participants sat in a dim-lit sound-proof test booth in front of a standard LCD screen, and a standard AZERTY French keyboard. Participants underwent first a Pre-Training phase in which they learnt the difference between target and composite faces, and between CW and CCW inclinations. Then, a Training phase in which they were presented with an RSVPs identical to the one of the main experiment, but with two modifications: 1) Targets were only Neutral Faces, 2) the stimuli duration were longer (467 ms), and decreased along the course of the 100 trials of the training until matching the target duration intended for the main task (117 ms). For the main task, participants responded by using their left hand, pressing the letters "A" and "Z" to indicate CCW or CW inclination. For the secondary task, they used their right hand to indicate the number of faces (either 1 or 2) on the numeric pad of the keyboard. Participants who did not reach an overall $70 \%$ accuracy rate on the Training set had to do it again, to a maximum of three times (all participants reached criterion after 2 rounds). Immediately after this phase, the first author delivered the hypnotic induction and administered the "emotional numbing" suggestion. Once the suggestion had been delivered, he instructed the participants to get ready for the main experiment, in which they would have to execute the same two tasks as before, as fast and as accurately as possible, while under the effect of the suggestion. The experimenter then left the room. There were six blocks of 100 trials each, separated by 3 minute pauses. During the pauses, the experimenter reinforced the hypnotic suggestion (see Appendix B in Supplementary Methods for the complete hypnosis procedure). Participants of the No Hypnosis group did not receive any kind of induction procedure or suggestion, but were instructed to use the pauses to close 
their eyes, relax, and hone their concentration in order to perform to the best of their abilities.

\subsection{Post-testing}

Since participants in the Hypnosis Group were exposed to a hypnotic suggestion that alluded indirectly to the emotional value of the targets, they were debriefed after the experimental task to control for suspicion of hypothesis. The debrief was also used to probe for any anomalous or unwanted behaviors elicited by poor understanding of the suggestion, or by convoluted expectations regarding the hypnosis procedure (e.g., "Hypnosis made me fall asleep completely" or "Hypnosis changed the way I controlled my hands"). No participant manifested any of the latter.

\section{RESULTS}

\subsection{Statistical analyses}

Our main goal was to evaluate if our paradigm would elicit an $A B$ susceptible to the ASE, and if so, how would hypnosis interrupt or attenuate the influence of the latter over the former. To this end, we decided to focus on the accuracy scores for the main task. Response times were included in the analysis, but only for developing the drift

diffusion models. See Statistical Analyses in Appendix A: Extended Methods for a detailed account on how all analyses were performed.

Accuracy was modeled by implementing (generalized) linear mixed models, with a random intercept per participant. We chose a hierarchical modeling approach in order to account for individual differences and for imbalances in sample sizes across factors and levels. We performed significance tests by means of likelihood ratio tests that 
compared our models to simpler models, in which the relevant predictor was removed (null model). ANOVA tables were computed through Analysis of Deviance (Type II Wald $X^{2}$ test), and post-hoc pairwise comparisons through contrasts of least-squares means setting a $0.95 \mathrm{Cl}$, then Bonferroni-corrected.

We also implemented Ratcliff's drift-diffusion model (1978) to analyze response time distributions through sequential sampling for correct and incorrect trials. The model's parameters were estimated through the maximum likelihood method using the fast-dm software (Voss, Rothermund \& Voss, 2004). The resulting $v$, a and t0 parameters were then modeled through mixed models, utilizing the same regressors as for the model for accuracy, a random intercept per participant and the same modeling and statistical procedures.

We conducted simulation-based a priori and observed power analyses. For a priori simulation-based analyses, we assumed that all conditions would have the same amount of participants, and produced 1000 cohorts starting at $n=7$ per condition, and then raised the $\mathrm{n}$ in steps of 2 until assessing a scenario in which $\mathrm{n}$ per condition was equal to 34 participants (i.e., 1000 simulations with $n=7$ participants per condition, 1000 simulations with $\mathrm{n}=9$, and so on). Following our hypothesis, we considered that differences in mean accuracy between Angry and Neutral T2 trials would be maximal for Hypnotized Lows, intermediate for Hypnotized Mediums and minimal for Hypnotized Highs. Based on previous observations (de Jong, Koster \& Martens, 2009), we estimated these differences as $10 \%, 5 \%$ and $1 \%$ respectively (SD=7\%). For nonhypnotized participants, we simulated two scenarios, a neutral one in which the effect of Anger Superiority would be even across groups, and a hypothesis-driven one in which the effect would depend on hypnotizability even in the absence of a hypnotic procedure. The simulations showed that for detecting a Hypnosis $\mathrm{x}$ Hypnotizability $\mathrm{x}$ 
T2Emotion interaction with an alpha $=0.05$ and a power equal or greater than $80 \%$, a minimum of $n=13$ per condition was required.

Simulation-based replication of observed power for groups with the same summary characteristics as those observed we collected experimentally (same n, same mean, same SD per condition) indicated a power of $91 \%(95 \% \mathrm{Cl}=90,93)$ to detect the same significant Hypnosis x Hypnotizability x T2 interaction for alpha $=0.05$.

Effect sizes were estimated by calculating Cohen's $d$ at each contrast, correcting for sample size $n<20$. Following this computation, $a d=0.2$ is interpretable as a small effect, $d=0.5$ as a medium effect and $d=0.8$ as a large effect.

\subsection{Attentional Blink, Emotional Salience \& Hypnotic Intervention}

We first plotted the accuracy scores across lags (see Fig.2), revealing the presence of a main blink effect in all groups and conditions. This blink was modulated by the emotional value of T1 and T2: performance was best when T2 was angry, and when it was neutral, performance depended on the emotional salience of T1. 


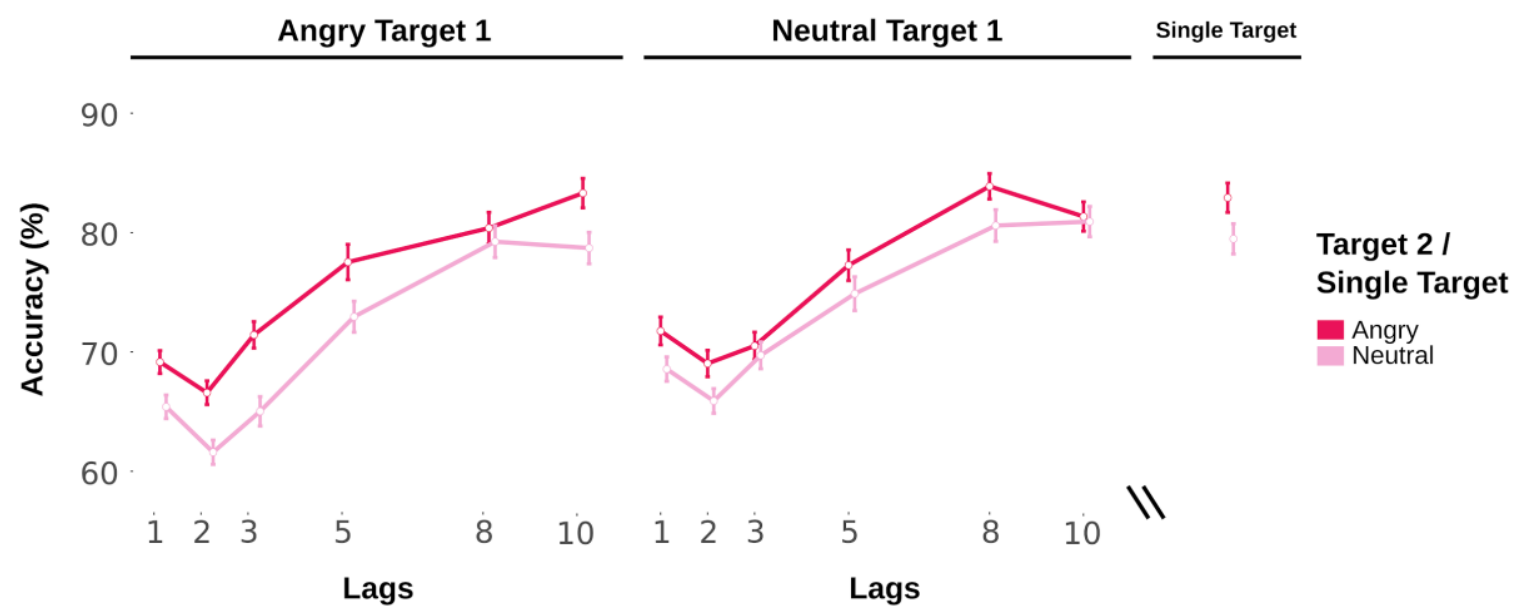

Figure 2: Attentional Blink and Anger Superiority, reflected by performance on the FaceOrientation Task. Accuracy scores (percentage correct) for all double and single-target trials, across all lags, for all conditions. Error bars: Standard Error.

Neither the hypnotic procedure nor participant hypnotizability interacted significantly with the emotional value of T1 (see Figure S3 in Appendix A, Additional Supplementary Figures). However, the hypnotic procedure successfully attenuated T2's emotional impact on accuracy as a function of hypnotizability, in a manner coherent with the content of the hypnotic suggestions. These effects were analyzed from two different perspectives: across all lags (Fig. 3A), and for lags collapsed into Early and Late Lag categories (Fig. 3B) $)^{3}$.

3 The pre-existing literature on face-based attentional blink (de Jong et al., 2009; de Jong et al., 2007) confirms that "Lag 1 sparing" is either negligible or non-existant for these kinds of paradigms. Rather, performance tends to be similar amongst lags affected by the AB's bottleneck. This is also the case for late lags, which usually plateau after a certain degree. Since we observed the same pattern in our data, and we scouted differences between early and late lags that could be of theoretical interest, we created the categories "Early" (lasg 1, 2, 3) and "Late" (lags 5, 8, 10). 
We observed that hypnotized Lows exhibited an ASE, in the form of increased performance for angry T2 trials with respect to neutral T2 trials, for both early and late trials (Accuracy Difference Angry minus Neutral T2 Early lag trials $=7 \%(95 \% \mathrm{Cl} 3.3$, 10.7), Cohen's $d=0.85$; Late Lags: $5 \%(0.9,9.1), d=0.5)$. Hypnotized Mediums exhibited the same increase, but only for early lags (Early Lags: $5 \%(1.6,8.4), d=0.73$; Late Lags: $1 \%(-3.9,4.9), \mathrm{d}=0.11)$. Hypnotized Highs only presented negligible differences across lags (Early Lags: $2 \%(-1.5,5.5), d=0.25$; Late Lags: $3 \%(-1.2,7.2)$, $\mathrm{d}=0.26$ ). For the Non-hypnotized group, Lows and Mediums did not perform better in angry T2 trials over neutral T2 trials (Lows: $1 \%(-2.6,4.6), d=0.12 ; 0 \%(-4.4,4.4), d=0$; Mediums: $1 \%(-2.3,4.3), d=0.13 ; 3 \%(-0.9,6.9), d=0.28)$. Highs did, only on early lags $(7 \%$ (3.3, 10.7), $d=0.88$

$1 \%$ $d=0.01)$.

A.
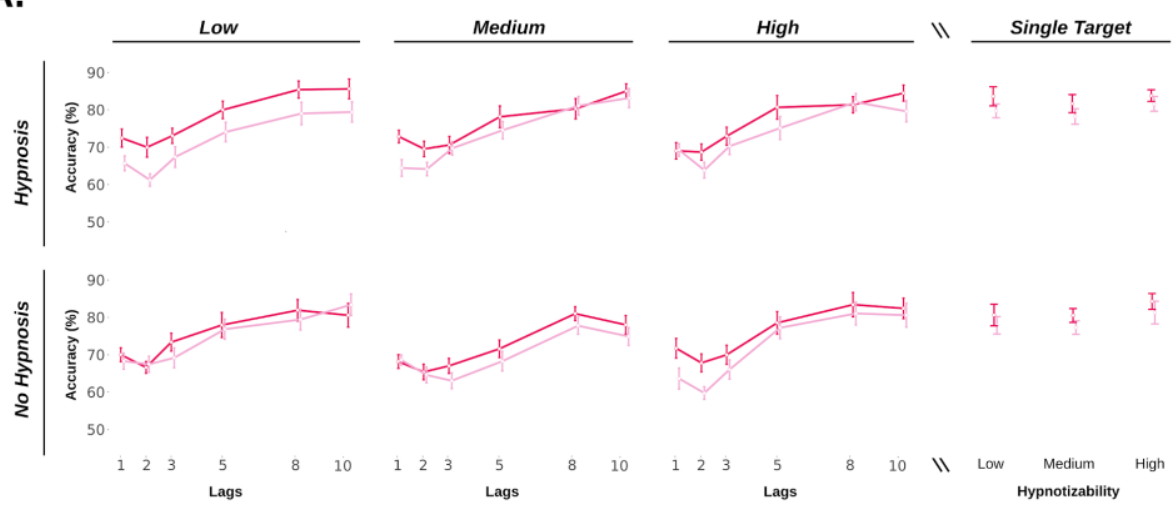

Target 2 Single Target Angry

B.
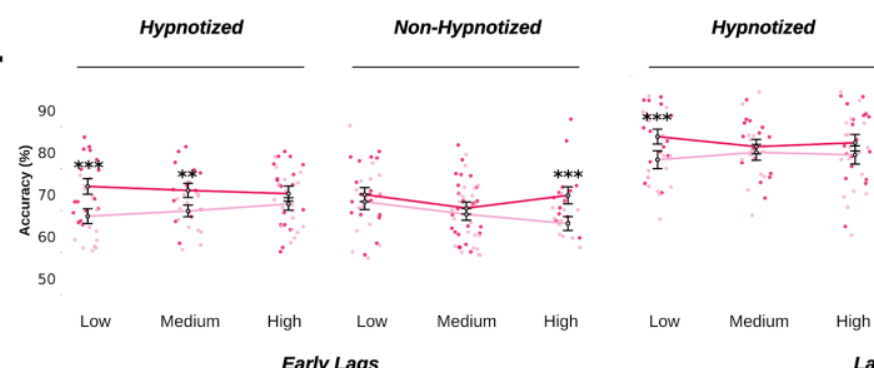

Non-Hypnotized

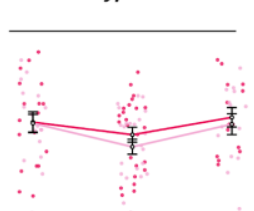

Early Lags

Figure 3: The Anger Superiority effect, modulated by the hypnosis procedure. A. Performance differences between Angry T2 and Neutral T2 trials, across all lags. B. Performance differences between 
Angry T2 and Neutral T2 trials, for Early Lag and Late Lag categories. Error bars: Standard Error; posthoc contrast $p$-values were Bonferroni-corrected; Cohen's $d$ was corrected for $n<20$; dots represent participants' means.

We tested the statistical significance of all these observations through the model "Accuracy $\sim$ Lag Category $\times \mathrm{T} 1_{\text {emotion }} \times \mathrm{T} 2_{\text {emotion }} \times$ Hypnotizability $\times$ Hypnotized $+\varepsilon$ ". The regressor Lag Category was a 2-level categorical factor (Early Lags, Late Lags). $\mathrm{T} 1_{\text {emotion }}$ and $\mathrm{T} 2_{\text {emotion }}$ were 2-level categorical factors (Angry, Neutral). Hypnotizability was a 3-level categorical factor (Low, Medium, High), and Hypnotized was a 2-level categorical factor (Hypnotized, Non-Hypnotized).

Our model presented main effects of Lag Category $\left(x^{2}=763, D F=1, p<0.0001\right), T 1_{\text {emotion }}$ $\left(X^{2}=27, D F=1, p<0.0001\right)$ and $T 2$ emotion $\left(X^{2}=29, D F=1, p<0.0001\right)$, together with a T1 $x$ $T 2$ interaction $\left(X^{2}=70, D F=1, p=0.001\right)$. These effects confirmed the observed presence of the $A B$, the ASE produced by each of the targets, and their interaction. Our model also presented a 3-way interaction T2emotion $x$ Hypnotizability $x$ Hypnotized $\left(X^{2}=14\right.$, $\mathrm{DF}=2, \mathrm{p}=0.001$ ) and a 4-way interaction $\mathrm{T} 2$ emotion $\mathrm{x}$ Lag Category $\mathrm{x}$ Hypnotizability $\mathrm{x}$ Hypnotized, $\left(X^{2}=7, D F=2, p=0.03\right)$. This was consistent with the observed effects of hypnosis on the ASE for T2. For a statistical summary, see Table 1.

\section{Table 1}

\section{Model: Accuracy (\%) $\sim$ Lag Category X T1 emotion X T2 emotion $_{\text {X }}$ Hypnotizability X Hypnotized $+\varepsilon$}

$\begin{array}{llll} & \mathbf{x}^{2} & \mathrm{DF} & \operatorname{Pr}\left(>\mathbf{x}^{2}\right) \\ \text { Laq Cateqorv } & 763 & 1 & <0.0001 \\ \text { T1 emotion } & 27 & 1 & <0.0001 \\ \text { T2 }_{\text {emotion }} & 70 & 1 & <0.0001\end{array}$




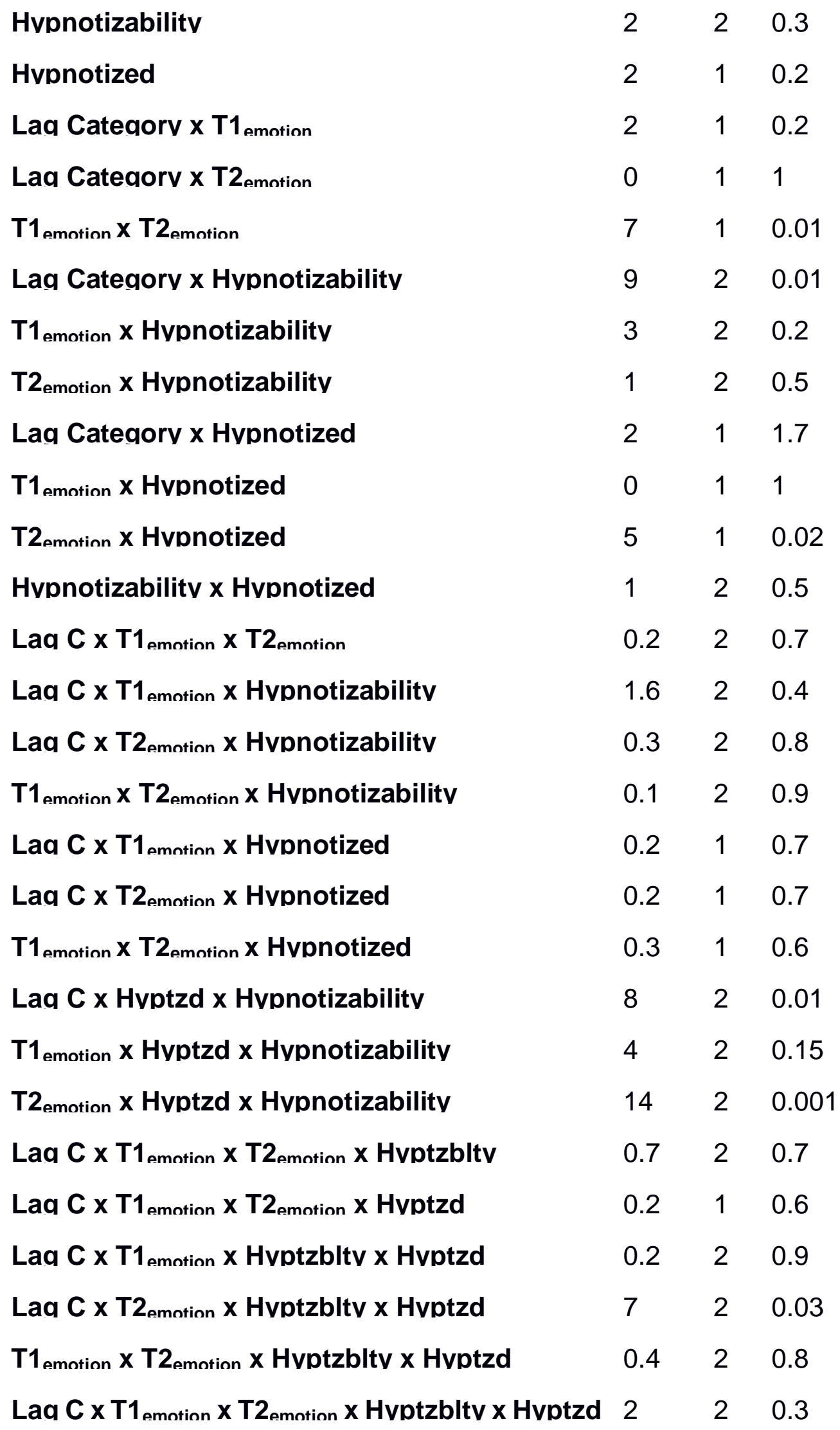


Table 1: Analysis of Deviance (Type 2 Wald $X^{2}$ test) for the model Accuracy $\sim$ Lag

Category $X \mathrm{~T} 1_{\text {emotion }} \mathrm{X} \mathrm{T} 2_{\text {emotion }} \mathrm{X}$ Hypnotizability $\mathrm{X}$ Hypnotized $+\varepsilon \cdot \mathrm{X}^{2}$ : chi-square; $D F$ : Degrees of Freedom; $\operatorname{Pr}\left(>X^{2}\right)$ : probability of obtaining the target chi-square statistic given that the null hypothesis is true.

In sum, we found no evidence that $\mathrm{T} 1$ processing depended on hypnosis or hypnotizability. However, we did find a significant interaction between hypnosis, hypnotizability and T2 processing. Accuracy differences between Neutral and Angry faces in T2 position were minimal for Highs under hypnosis (Post-hoc pairwise contrast between Angry $\mathrm{T} 2$ and Neutral $\mathrm{T} 2$ in Early Lags: $x^{2}=3.6, D F=1, p=0.7$; in Late Lags: $\left.X^{2}=6.25, D F=1, p=0.14\right)$. For Lows, on the other hand, the ASE was strongly present (Early: $x^{2}=26, D F=1, p<0.0001$; Late: $x^{2}=19, D F=1, p<0.0001$ ). Mediums fell "in between", as the advantage of angry faces was still present at early lags $\left(X^{2}=12, D F=1\right.$, $p=0.007)$, but no evidence was found for it at late lags $\left(X^{2}=1, D F=1, p=1\right)$. Without hypnosis, we found no evidence for the advantage of angry faces at either lag category for Lows and Mediums (Lows Early $X^{2}=1, D F=1, p=1$; Late: $X^{2}=0, D F=1, p=1$; Medium Early: $\mathrm{X}^{2}=1, \mathrm{DF}=1, \mathrm{p}=1$; Late: $\mathrm{X}^{2}=7, \mathrm{DF}=1, \mathrm{p}=.12$ ). However, we did find evidence for anger superiority in Highs at early lags $\left(\right.$ Early: $\chi^{2}=20, D F=1, p<0.0001$; Late: $x^{2}=1$, $\mathrm{DF}=1, \mathrm{p}=1)^{4}$.

\subsection{Drift diffusion analysis}

In order to better interpret the effects of the hypnotic procedure, we implemented a drift-diffusion decision model to analyze response time distributions through sequential

$4 \quad$ All post-hoc pairwise contrasts were Bonferroni-corrected. 
sampling, for correct and incorrect trials. When inspecting response times prior to running the model, we found that regardless of target emotion and lag, hypnotized participants produced faster responses the higher their hypnotizability (median RT in ms, Lows $=806(S D=282)$, Mediums $=650(207)$, Highs $=588(177))$. This was not the case for non-hypnotized participants (median RT in ms, Lows = 655 (206), Mediums = 773 (243), Highs = 672 (237)). See Table SM1 Appendix A, Supplementary Tables for Response Time statistical modelling).

Drift-diffusion models of the kind we implemented (Ratcliff, 1978; Voss, Rothermund \& Voss, 2004) present three main parameters: $a$ is the threshold for decision, and thus an indicator of the response strategy. Generally, a lower a corresponds to a more liberal response, and higher a to a more conservative, cautious response strategy. The drift rate $v$ is the rate of information accumulation, and thus essentially equivalent to the $d^{\prime}$ in Signal Detection Theory. The non-decision time to represents all other psychological and physiological durations not pertaining to the decision at hand, including encoding and execution. Of these three parameters, $a$ and to were susceptible to interactions with Hypnotizability for participants who had been hypnotized ${ }^{5}$. As shown in Figure 4, mean a decreased with the increase in hypnotizability for hypnotized participants, pointing to a direct correlation between hypnotizability and lower decisional thresholds, typically associated with cognitive control (mean a for Hypnotized Lows=1.8 (SD=0.3); Mediums=1.8 (0.3), Highs=1.8

$5 \quad$ As stated above, a (decisional threshold) and to (non-decisional response time) were the only parameters that changed with hypnotizability for participants who were under the effect of hypnotic suggestion. However, changes linked to hypnotizability that are independent of hypnotic induction and suggestion cannot be attributed to the process of hypnosis (Terhune et al., 2017). For the full extent of interactions pertaining $a, v$ and to, please see Supplementary Methods, Appendix A, Table SM. 3) 
(0.3); Non-Hypnotized Lows=1.85 (0.3); Mediums=1.63 (0.2), Highs=1.47 (0.3)). On the other hand, to was significantly larger for Medium Hypnotized participants during Late Lag trials (mean t0 for Hypnotized participants during Early Lags $=0.19(0.09)$, during Late Lags $=0.25(0.11))$.

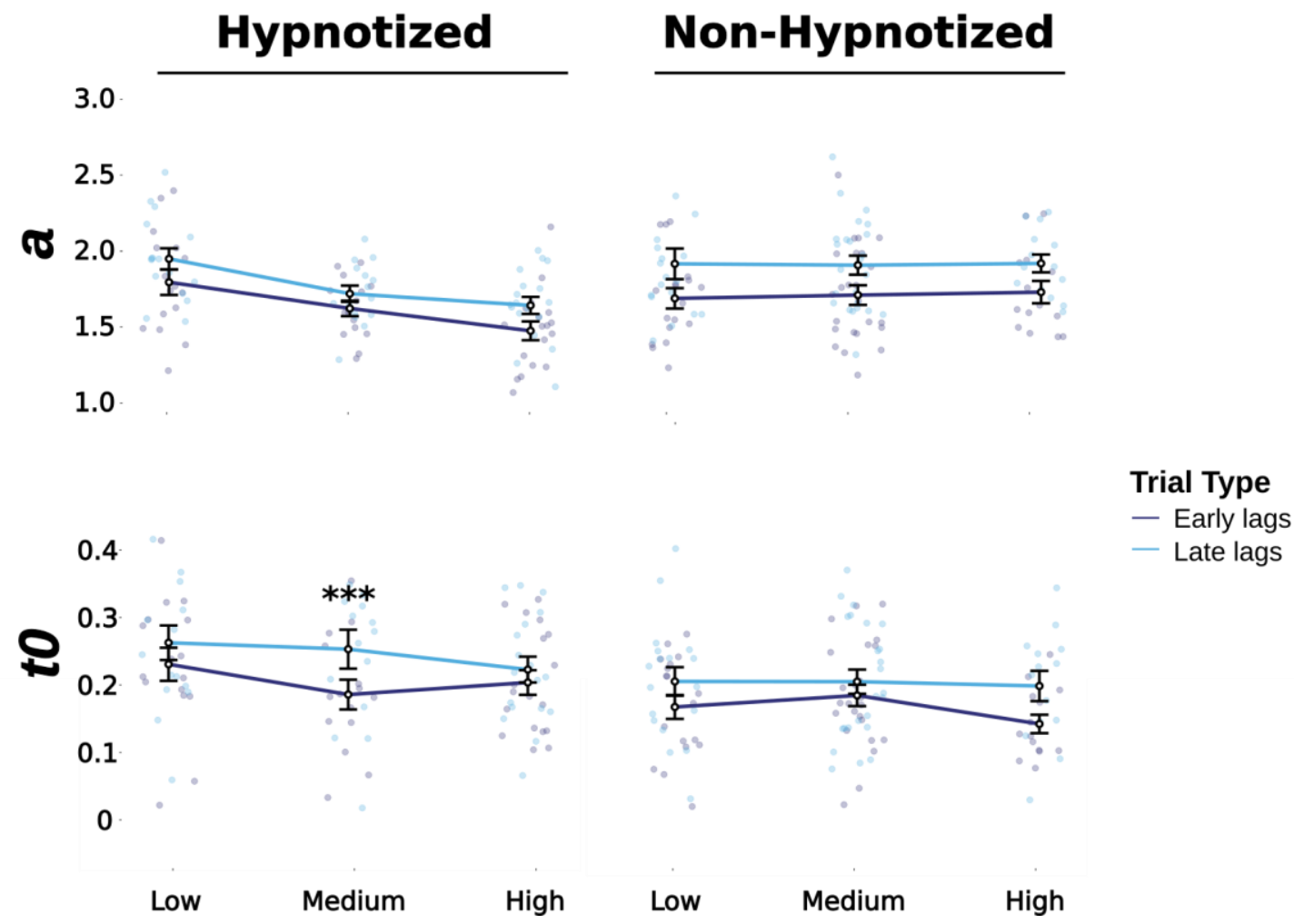

Figure 4: Evolution of response bounds and non-decisional time in a drift diffusion model across hypnotizability. Error bars: Standard Error; post-hoc contrast p-values were Bonferroni-corrected; dots represent participants' means.

We tested the statistical significance of this effect by modelling $a$, t0 and $v$ against the same predictors as the previous models. For the a parameter we observed a significant Hypnotizability $x$ Hypnotized interaction $\left(X^{2}=6, D F=2, p=0.04\right)$. By repeating the model for the hypnotized participants alone, we found a Hypnosis main effect $\left(X^{2}=14, D F=2\right.$, $p=0.0009)$, which was not significant for the non-hypnotized participants $\left(x^{2}=0.04\right.$, 
$\mathrm{DF}=2, \mathrm{p}=1)$. For the to parameter we observed a significant interaction Lag Category $x$ Hypnotizability $x$ Hypnotized $\left(x^{2}=14, D F=2, p=0.0007\right)$. We observed that this effect was mainly driven by Hypnotized Mediums, who sported a t0 difference between Early Lag and Late Lag trials $\left(X^{2}=29, D F=1, p<0.0001\right.$; post-hoc contrast, Bonferronicorrected). See Table SM3 in Appendix A, Supplementary Tables for a full summary of these effects.

\subsection{Target-counting task}

The secondary task (counting target faces amongst distractors) served the auxiliary purpose of ensuring that participants would sustain their attention throughout the entire RSVP, a condition necessary for the elicitation of the blink. We observed that accuracy scores for this task were similar for all hypnotizabilities, for hypnotized and nonhypnotized participants alike (percentage correct for Hypnotized: Highs 77\%, Mediums 79\%, Lows 77\%; Non-Hypnotized: Highs 76\%, Mediums 73\%, Lows 78\%; interaction Hypnotized $x$ Hypnotizability $x^{2}=3, D F=2, p=0.2$ ). We also observed that including participants who had not properly counted both targets did not change the final pattern of results for the main task. The complete account of effects and interactions for this task can be found on Table SM2 in Appendix A, Supplementary Tables. 


\section{DISCUSSION}

The present work was developed to find out where and how along the decision process hypnosis intervenes, and how its effects depend on the time course of attention. Our findings were twofold. We observed that hypnosis reduced the ASE for High participants, but only for the second target in the visual stream (T2). We also found that non-hypnotized Highs displayed an ASE, but Mediums and Lows did not.

The mechanisms behind the ASE remain a matter of discussion (Frischen, Eastwood \& Smilek, 2008). Several accounts consider it an automatic bottom-up process (Öhman, 2002; Öhman, Flykt, Lundqvist, 2001), but numerous studies have exposed its vulnerability to top-down effects (de Jong, Koster \& van Wees, 2009; Stein, Peelen, Funk \& Seidl, 2010; Yao, Ding, Qi \& Yang, 2014). Our results fall in line with this second trend, by showing the ASE can be attenuated through top-down hypnotic modulation. This "numbing effect" was not just a global subjective phenomenon; rather, it was evidenced by performance changes in the execution of a task unrelated to the appraisal of facial emotion. Remarkably, Highs were permeable to hypnotic effects even when suggestion content did not target task-relevant stimuli features. This entails that the hypnotic modulation of a global (emotional) state of mind can successfully propagate to lower-level appraisal mechanisms. 
The potential reasons why T1 was spared by the hypnotic procedure are numerous and not exclusive. It is unclear whether the ASE results from a cognitive emotional appraisal process, a purely perceptual bias favoring emotional facial features, or a combination of both (Frischen, Eastwood, \& Smilek, 2008; Mermillod, Vermeulen, Lundqvist \& Niedenthal 2009). Since the processing dynamics of T1 and T2 differed in terms of task execution and temporal attention constraints, maybe the low-level perceptual and high-level appraisal components of the ASE contributed differently to targets' salience. At each trial, participants had to scan every stimulus in the visual stream to count the targets and then indicate the inclination orientation for the last perceived target. Namely, after spotting T1, participants had to count it as one target, evaluate its inclination, commit the information to memory and rapidly switch their focus towards the subsequent stimulus. In contrast, T2's late processing was unconstrained, and led directly to response preparation (Marti, Sigman \& Dehaene, 2011; Lagroix, Di Lollo \& Spallek, 2018). Because of these differences, it is possible that T2's ASE relied more on high-order affective appraisal, while T1's hinged mainly on low-level perceptual advantage. If hypnosis propagated down to the affective appraisal level, but no further, this would explain the sparing of $\mathrm{T} 1$ emotional salience.

Trials constrained by the $A B$ often exhibit identical sensory processing as unencumbered trials, but delayed (or even absent) frontal cortex activation (Marti, Sigman \& Dehaene, 2011). This AB effect on attention-specific downstream frontal functions would be coherent with an effect on response preparation. If hypnosis effects were restricted to a time after encoding, i. e. to the response preparation stage (Sigman \& Dehaene, 2005), then less-constrained lags where response preparation was less disturbed would necessarily be more affected by it. Our results confirm this premise: 
hypnotized Mediums and Highs displayed smaller performance differences for smaller blinks (i. e., Late Lags).

Our diffusion model findings also strengthen the case for hypnotic effects being tied to response processes. Changes in response thresholds that do not harm performance can be associated with optimality-driven control strategies (Bogacz, Brown, Moehlis, Holmes \& Cohen, 2006). Of the three main parameters of the model, "a" is the only one associated directly to the development of response strategies (Voss, Rothermund \& Voss, 2004). Here, in hypnotized participants "a" decreased as hypnotizability rose, and as performance differences decreased. This idea of hypnosis-driven control strategies intervening with response processes is consistent with the findings by Augustinova \& Ferrand (2012), who proposed hypnotic effects intervened within the range of response competition. Neuroimaging research contributes to this conclusion as well, implicating the dorsolateral prefrontal and anterior cingulate cortices as recurrent participants of multimodal hypnotic responding (Landry \& Raz, 2015; Jiang, White, Greicius, Waelde \& Spiegel, 2016).

Our second set of findings was related to the salience of T2 outside hypnosis. Highs exhibited an ASE at early lags while not hypnotized, but Lows did not. Previous research has shown that the impact of emotional salience on performance depends largely on whether emotion belongs in the attentional task-set (Stein, Funk \& Seidl, 2010). Thus, our results could indicate attentional task-set differences between hypnotized and non-hypnotized participants.

In our paradigm, the emotional valence of targets was task-irrelevant by design: instructions were delivered without alluding to targets' facial expressions, and training was conducted on an "all-neutral-faces" version of the paradigm. By construction, 
participants in the "no-hypnosis" condition never heard any reference to emotion. In light of this, it is possible that our hypnotic procedure had the "instruction-like" effect of updating the attentional task-set by introducing target emotion into task context. Our results could then be explained as participants responding through an updated taskset, producing responses that depended on hypnotizability. Lows, who ignored T2 emotional features when not hypnotized, found themselves under their influence after hypnosis introduced them into the task set. Highs found themselves in the same situation, but produced the opposite response. They successfully attenuated anger superiority as prescribed during hypnosis, but their increased susceptibility rendered them unable to ignore emotional salience despite it being task-irrelevant for the No Hypnosis condition (a lack in cognitive control consistent with previous findings; see Terhune \& Cardeña, 2014). These theoretical conclusions would require further validation; here, we simply suggest that while hypnotizability as a personality trait is yet not fully understood (Terhune, Cleeremans, Raz \& Lynn, 2017; Terhune \& Cardeña, 2014), Highs could be more inclined to engage in the appraisal of facial emotion as a social suggestion regardless of relevance (Colloca \& Benedetti, 2009; Wickramasekera \& Szlyk, 2003).

Taken together, we posit that our results argue for interpreting hypnotic effects in terms of response control, conditional to the availability of temporal attention. Furthermore, our results portray hypnotic response as the result of a two-tier process. We propose that hypnosis first catalyzes an alteration of the task-set for participants of all hypnotizabilities; then, depending on their hypnotizability and provided there is enough time to displace attention, participants may enact the actual content of the suggestion, producing a hypnotic response. This model would be compatible with predominating views of hypnosis as top-down control (Terhune, Cleeremans, Raz \& Lynn, 2017), and 
help disentangle hypnotic responding from the attentional non-hypnotic effects of the hypnosis procedure.

\section{AUTHOR CONTRIBUTIONS}

Hernán Anlló designed the experiment, prepared the stimuli, prepared the hypnotic induction and suggestions, administered the test to participants, analyzed the data, elaborated the conclusions, wrote the manuscript.

Joshua Hagège collaborated with study design and stimuli preparation, administered the test to participants in the pilot stage.

Jérôme Sackur provided supervision at every stage of the process, contributed designing the experiment, contributed in the interpretation of results and the elaboration of conclusions, corrected the manuscript.

\section{ACKNOWLEDGEMENTS}

We thank Isabelle Brunet for the recruitment. We thank Dr. Pascal Mamassian, Dr. Catherine Tallon-Baudry and Dr. Katsumi Watanabe for their invaluable contributions. We are grateful to all participants of this study.

\section{DATA AVAILABILITY}

Data is licensed for non-commercial use only, and has been made available for download at the OSF repository of the project (XXXXXXXXXX). Please address any questions to hernan.anllo@cri-paris.org

\section{FUNDING}


This project was funded by a fellowship from Frontieres du Vivant (CRI) to Hernán Anlló. It was supported by grants from the Région Ile-de-France, Fondation de France, LabEx IEC (ANR-10-LABX-0087), IdEx PSL (ANR-10-IDEX-0001-02). Later stages were supported by Grants-in-Aid for Scientific Research (17F17008, 17H00753) and a Postdoctoral Fellowship for Overseas Researchers from the Japan Society for the Promotion of Science (JSPS), and CREST (JPMJCR14E4). The authors report no conflict of interest.

\section{REFERENCES}

Arnell, K. M., Killman, K. V., \& Fijavz, D. (2007) Blinded by emotion: Target misses follow attention capture by arousing distractors in RSVP. Emotion, 7, 465-477.

Augustinova, M., \& Ferrand, L. (2012). Suggestion does not de-automatize word reading: Evidence from the semantically based Stroop task. Psychon Bull Rev, 19(3), 521-527. doi: 10.3758/s13423-012-0217-y

Bogacz R., Brown E., Moehlis J., Holmes P., Cohen J. (2006) The physics of optimal decision making: a formal analysis of models of performance in two-alternative forcedchoice tasks. Psychol Rev. Oct; 113(4): 700-765. doi: 10.1037/0033-295X.113.4.700

Bryant R., Kapur A., (2006) Hypnotically induced emotional numbing: The Roles of Hypnosis and Hypnotizability Intl. Journal of Clinical and Experimental Hypnosis, 54(3): 281-291. DOI: 10.1080/00207140600689462. 
Chun M., Potter M. (1995) A two-stage model for multiple target detection in rapid serial visual presentation. J Exp Psychol Hum Percept Perform 2: 109-127. doi: 10.1037/0096-1523.21.1.109

Colloca, L., \& Benedetti, F. (2009). Placebo analgesia induced by social observational learning. Pain, 144(1- 2), 28-34. doi: 10.1016/j.pain.2009.01.033

de Jong P.J., Koster E.H.W., van Wees R., Martens S. (2009) Emotional facial expressions and the attentional blink: Attenuated blink for angry and happy faces irrespective of social anxiety. Cogn Emot 23: 1640-1652. doi:10.1080/ 02699930802490227.

de Jong P.J., Martens S. (2007) Detection of emotional expressions in rapidly changing facial displays in high and low-socially anxious women. Behav Res Ther 45: 12851294. doi:10.1016/j.brat.2006.10.003.

Elman, D. (1984) Hypnotherapy. Westwood Publishing Co ,U.S.

Frischen, A., Eastwood, J. D., \& Smilek, D. (2008). Visual search for faces with emotional expressions. Psychological Bulletin, 134, 662-676. doi:10.1037/ 00332909.134.5.662

Hansen C., Hansen R. (1988) Finding the Face in the Crowd: An Anger Superiority Effect. Finding the Face in the Crowd: An Anger Superiority Effect. Journal of Personality and Social Psychology 54(6):917-24. DOI: 10.1037/0022-3514.54.6.917

Jiang, H., White, M. P., Greicius, M. D., Waelde, L. C., \& Spiegel, D. (2016). Brain activity and functional connectivity associated with hypnosis. Cerebral Cortex. doi: 10.1093/cercor/bhw220 
Landry, M., \& Raz, A. (2015). Hypnosis and imaging of the living human brain. American Journal of Clinical Hypnosis, 57(3), 285-313. doi: $10.1080 / 00029157.2014 .978496$

Lagroix H., Di Lollo V., Spalek T. (2018) The attentional blink: why does Lag-1 sparing occur when the dependent measure is accuracy, but Lag-1 deficit when it is RT? Psychological Research. https://doi.org/10.1007/s00426-018-1026-3

Maratos F., Mogg K., Bradley B., (2008) Identification of angry faces in the attentional blink December. Cognition and Emotion 22(7):1340-1352 DOI:

$10.1080 / 02699930701774218$

Marti S., Sigman M., Dehaene S. (2011) A shared cortical bottleneck underlying Attentional Blink and Psychological Refractory Period. Neuroimage Vol 59, Issue 3, 2883-2898. doi:10.1016/j.neuroimage.2011.09.063

Martin, J. R., \& Pacherie, E. (2019). Alterations of agency in hypnosis: A new predictive coding model.Psychological review, 126(1), 133.

McHugo M, Olatunji B., Zald D. (2013) The emotional attentional blink: what we know so far. Frontiers in Human Neuroscience, Volume 7 , Article 151. doi: 10.3389/fnhum.2013.00151

Mermillod M., Vermeulen N., Lundqvist D., Niedenthal P. (2009) Neural computation as a tool to differentiate perceptual from emotional processes: The case of anger superiority effect. Cognition 110 (2009) 346-357. doi:10.1016/j.cognition.2008.11.009

Most, S. B., Smith, S. D., Cooter, A. B., Levy, B. N., Zald, D. H. (2007). The naked truth: Positive, arousing distractors impair rapid target detection. Cognition \& Emotion, 21, 964-981. 
Nieuwenstein M., Potter M., Theeuwes J. (2009) Unmasking the attentional blink. J

Exp Psychol Hum Percept Perform. 2009 February ; 35(1): 159-169. doi:10.1037/0096-1523.35.1.159.

Olivers C., Nieuwenhuis S. (2006) The Beneficial Effects of Additional Task Load, Positive Affect, and Instruction on the Attentional Blink. Journal of Experimental Psychology, Human Perception and Performance, Vol. 32, No. 2, 364-379. DOI: $10.1037 / 0096-1523.32 .2 .364$

Öhman, A. (2002). Automaticity and the amygdala: Nonconscious responses to emotional faces. Current Directions in Psychological Science, 11, 62-66.

Öhman, A., Flykt, A., Lundqvist, D. (2001). Unconscious emotion: Evolutionary perspectives, psychophysiological data and neuropsychological mechanisms. In R. D. Lane \& L. Nadel (Eds.), Cognitive neuroscience of emotion (pp. 296-327). New York, NY: Oxford University Press.

Ratcliff, R. (1978). A theory of memory retrieval. Psychological Review, 85, 59-108.

Raymond, J., Shapiro, K., Arnell, K. (1992) Temporary suppression of visual processing in an RSVP task: An attentional blink? Journal of Experimental Psychology: Human Perception \& Performance, 18, 849-860.

Sakai K., (2008) Task Set and Prefrontal Cortex. Annual Review of Neuroscience. Vol 31, Issue 1, 219-245. 10.1146/annurev.neuro.31.060407.125642

Sergent C., Baillet S., Dehaene S. (2005) Timing of the brain events underlying access to consciousness during the attentional blink. Nature Neuroscience. 8 (10):1391-1400. 
Sigman M., Dehaene S. (2005) Parsing a Cognitive Task: A Characterization of the Mind's Bottleneck. PLoS Biology, Vol 3., Issue 2, 335-349. 10.1371/journal.pbio.0030037

Smith S. D., Most S. B., Newsome L. A., Zald D. H. (2006). An emotion-induced attentional blink elicited by aversively conditioned stimuli. Emotion, 6, 523-527.

Stein T., Peelen M., Funk J., Seidl K. (2010). The Fearful-Face Advantage Is Modulated by Task Demands: Evidence From the Attentional Blink. Emotion, Vol. 10, No. $1,136-140$. DOI: $10.1037 / \mathrm{a} 0017814$.

Terhune D. B., Cleeremans A., Raz A., Lynn S.J. (2017) Hypnosis and top-down regulation of consciousness. Neuroscience \& Behavioral Reveiws. doi: http://dx.doi.org/10.1016/..neubiorev.2017.02.002

Terhune D., Cardeña E. (2014) Hypnotizability, Personality Traits, and the Propensity to Experience Alterations of Consciousness. Psychology of Consciousness: Theory, Research, and Practice 2014, Vol. 1, No. 3, 292-307 http://dx.doi.org/10.1037/cns0000026

Thorpe, S., Fize, D., Marlot, C. (1996) Speed of processing in the human visual system. Nature, 381, 520-522.

Vogel, E., Luck, S., Shapiro, K. (1998) Electrophysiological evidence for a postperceptual locus of suppression during the attentional blink. Journal of Experimental Psychology: Human Perception \& Performance, 24, 1656-1674.

Voss, A., Rothermund, K., \& Voss, J. (2004) Interpreting the parameters of the diffusion model: An empirical validation. Memory and Cognition, 32, 1206-1220. 
Wickramasekera, I. E., 2nd, \& Szlyk, J. P. (2003). Could empathy be a predictor of hypnotic ability? Int J Clin Exp Hypn, 51(4), 390-399.

Yao S.,Ding C., Qi S., Yang D. (2014) Value associations of emotional faces can modify the anger superiority effect: behavioral and electrophysiological evidence. SCAN 9, 849-856, doi:10.1093/scan/nst056

\section{SUPPLEMENTARY METHODS}

Appendix A - Extended methods, full statistical models and supplementary results

\section{Extended methods}

\subsection{Stimuli}

In order to produce these stimuli, first, we selected all available male, neutral-looking and angry-looking faces from the Cohn-Kanade (Kanade, Cohn \& Tian, 2000; Lucey, Cohn, Kanade, Saragih, Ambadar \& Matthews, 2010), AR Face (Martinez \& Benavente, 1998), ADFES (van der Schalk, Hawk, Fischer \& Doosje, 2011), NimStim (Tottenham, Tanaka, Leon, Nurse, Hare, Marcus, Westerlund, Casey \& Nelson, 2009), Chicago Face (Ma, Correll, Wittenbrink, 2015) and RaFD (Langner, Dotsch, Bijlstra, Wigboldus, Hawk \& van Knippenberg, 2010) databases as raw materials. All images were cropped and resized to 228 by 240 pixels. Faces were then converted to grayscale and manually cropped again to a 2.5 o major radius oval (using the tip of the nose as center). The resulting oval-shaped faces were sorted out by the first two authors, in order to select those that better represented anger and neutrality. 
We then used the SHINE Matlab Toolbox (Willenbockel, Sadr, Fiset, Horne, Gosselin \& Tanaka, 2010) to match mean luminance and contrast (i. e. the standard deviation of the luminance distributions) of all selected faces to a template. Said template was the composite face that resulted from averaging all pixel values for all 40 Angry and 40 Neutral Faces combined. Background luminance was set at a nominal 122 value of gray $\left(25 \mathrm{~cd} / \mathrm{m}^{2}\right)$. Then, in order to develop the composite faces stimuli in the same range of luminance, we combined all of the already matched neutral and angry faces into a single pool and used them as raw materials: each composite face was the result of averaging 25 faces selected at random from the pool. We produced 40 composite faces. Mean luminance of the stimuli was $18.5 \mathrm{~cd} / \mathrm{m}^{2}(\mathrm{SD}=2.21)$ for the Neutral Faces, $19 \mathrm{~cd} / \mathrm{m}^{2}(S D=1.95)$ for the Angry Faces and $18 \mathrm{~cd} / \mathrm{m}^{2}(S D=1.48)$ for the Composite Faces. Mean Michelson contrast was 0.77 ( $S D=0.01)$ for both Neutral and Angry Faces, and 0,76 (SD=0,03) for the Composite Faces.

\subsection{Participants}

Across the general population, High and Low susceptibility individuals each represent around 12 to 17 percent of the population (see Shor \& Orne, 1962; Anlló, Becchio \& Sackur, 2017). This distribution implies that even after exhaustive hypnotic susceptibility screenings, obtaining large samples for all hypnotizabilities can be a daunting task. For this reason, it was stipulated that recruitment would target as many people as possible, and continue until volunteers stopped responding to the call. Two calls were launched in parallel, independently of each other, by different research assistants. One was the call for hypnotic susceptibility screening: participation was voluntary, in exchange of university credit, for a 2-hour session of the Harvard Group Scale of Hypnotic Susceptibility (Shor \& Orne, 1962; Anlló, Becchio \& Sackur, 2017). The other was the call for the present experiment: participants were contacted 
by e-mail and recruited in several rounds, contingently on whether they had previously participated in the concurrent hypnotic susceptibility screenings, but without knowing that the experiment itself would involve hypnosis.

Participants from the Hypnosis Group were tested blindly, without the hypnosis practitioner knowing their level of hypnotizability at the time of explaining the task instructions or administering the hypnosis procedure. They were told that they would be taking part in an experiment that would include their response to hypnotic suggestion, and warned that all levels of susceptibility and hypnotic responding were relevant for the study. They were informed that their response, if any, to the procedure had no impact on their monetary compensation. It was also clearly stated that the authors did not have any particular expectations about their performance or hypnotic response. Participants on the No Hypnosis Group were also tested blindly and received the same introduction to the task, but did not receive any information whatsoever regarding the connection between the experiment and hypnosis. The mention of hypnosis and hypnotic susceptibility were explicitly avoided at all times throughout the testing of this group.

\section{$\underline{1.3 \text { Statistical analyses }}$}

\subsubsection{Generalized Linear Mixed Models}

We performed data analysis using $R$ ( $R$ Development Core Team, 2008). Accuracy was modeled by implementing (generalized) linear mixed models, with a random intercept per participant (Ime4; Bates, Maechler, Bolker \& Walker, 2015). We chose a hierarchical modeling approach in order to account for individual differences and for imbalances in sample sizes across factors and levels (Agresti, 2002; Jaeger, 2008). We performed significance tests by means of likelihood ratio tests that compared our models to simpler models, in which the 
relevant predictor was removed (null model) (Pinheiro \& Bates, 2000; Bolker, Brooks, Clark, Geange, Poulsen, Stevens \& White, 2008). ANOVA tables were computed through Analysis of Deviance (Type II Wald $\chi^{2}$ test), and post-hoc pairwise comparisons through contrasts of leastsquares means setting a $0.95 \mathrm{Cl}$, then Bonferroni-corrected (car and Ismeans $R$ packages; Fox \& Weisberg, 2011 and Lenth, 2016, respectively).

\subsubsection{Drift Diffusion Models}

We also implemented Ratcliff's (Ratcliff, 1978) drift-diffusion model to analyze response time distributions through sequential sampling for correct and incorrect trials. The model's parameters were estimated through the maximum likelihood method, using the fast- $d m$ software (Voss, Rothermund \& Voss, 2004); the resulting $v, a$ and tO parameters were then modeled through mixed models, utilizing the same regressors as for the model for accuracy, a random intercept per participant and the same modeling and statistical procedures as before.

\subsubsection{Observed and a-priori simulation-based power analyses}

Hypnotizability is a rather stable trait, with an almost normal/T-like population distribution (Shor \& Orne, 1963). This means that given a large enough sample, around 12 to $17 \%$ of the population will be found to be highly susceptible to hypnosis, another 12 to $17 \%$ will be of low susceptibility, and the remaining 70 to $76 \%$ will lie in between. This means that testing a group of highly susceptible individuals of as little as $n=20$ requires the face-to-face hour-long screening of roughly 200 people (in practice, these numbers are even higher, given screening errors and reluctance to continue the experiment after screening). For example, in the case of our work, 650 participants had to be screened, just to secure 105 individuals willing to 
continue with the actual experiment. This is extremely resource-consuming. Such conditions impose certain flexibility at the time of securing suitable sample sizes, particularly if, as we did, attempts are made to include the whole susceptibility spectrum into the experiment (a rare occurrence, mainly because of how resource-intensive this is; see Jensen et al, 2017).

Furthermore, because of our desire to manipulate the attentional task-set, in the present work it was of the utmost importance that participants had no prior knowledge of the paradigm and its stimuli at the time of performing the experiment. This obligated us to use an intergroup design: we recruited $3 \times 2$ groups of participants: High, Medium and Low susceptibility, with and without hypnosis. Once recruiting efforts were concluded, the Hypnosis Group was composed by 18 participants highly susceptible to hypnosis (Harvard score 9-12: Highs), 15 of medium susceptibility (score 5-8: Mediums) and 16 of low susceptibility (score $0-4$ : Lows). The No Hypnosis Group was composed of 14 Highs, 24 Mediums and 18 Lows. It should be noted that these numbers are in line with a sizable portion of existing hypnosis research, as well as many relevant papers on Attentional Blink, Anger Superiority Effects and Emotional Attentional Blink studying similar effects (see for example de Jong et al, 2007; Yao et al., 2014; Taylor et al., 2014; Stein et al., 2009; lani et al., 2009; Anderson, 2005).

In the present work we aimed at recruiting as many participants as financially and materially possible, and then, once recruitment was exhausted, we assessed if the sample we had gathered allowed for satisfactory statistical power. To evaluate obtained power without incurring in analyses that would be redundant with $p$-values and that would be easily adaptable to GLMMs, we used simulation-based power analysis.

For a-priori simulation-based power analysis, following our hypothesis, we considered that differences in mean accuracy between Angry and Neutral T2 trials would be maximal for 
Hypnotized Lows, intermediate for Hypnotized Mediums and minimal for Hypnotized Highs. Based on our previous observations, we estimated these differences as $10 \%, 5 \%$ and $1 \%$ respectively ( $S D=7 \%)$. Then, we assumed two possible behaviors for the control group, and performed two simulations. One, consistent with our hypothesis, in which hypnotizability of non-hypnotized participants was an important factor that would also come into effect as a marker of cognitive control, affecting accuracy despite the absence of hypnosis: here, we estimated that accuracy differences would be the opposite to those of hypnotized participants, namely $1 \%, 5 \%$ and $10 \%$ respectively $(S D=7 \%)$. This is because our hypothesis proposed that despite being task-irrelevant, the emotional value of targets would affect Highs because of their susceptibility, while sparing Lows. For the second scenario, we remained conservative, and assumed that non-hypnotized participants would behave as a standard control group: namely, the accuracy difference between Angry and Neutral trials would be the same for everyone, and the same as for the Low Hypnotized group (a strong Anger Superiority effect all around, of 10\%). This is because assuming regular behavior, we could estimate that hypnotizability would not influence Anger Superiority. It is worth noting that our observed data was consistent with the first scenario (and hence, our hypothesis), but to have an integral conservative power estimation we thought it necessary to conduct this second simulation as well.

The crucial point of these a-priori simulations was the $n$. We assumed that all conditions would have the same amount of participants, and produced 1000 cohorts starting at $n=7$, and then raised the $\mathrm{n}$ at each iteration in steps of 2 until assessing a scenario in which $\mathrm{n}$ per condition was equal to 34 participants (i.e., 1000 simulations with 6 groups $n=7,1000$ simulations with $\mathrm{n}=9$, and so on). Figure SM3 below shows that, always for alpha at 0.05 , the conservative 
simulation yielded estimated powers above $80 \%$ for groups of $n=13$ and above for detecting an interaction Hypnosis $x$ Hypnotizability $x$ T2 using the same model as with our data.

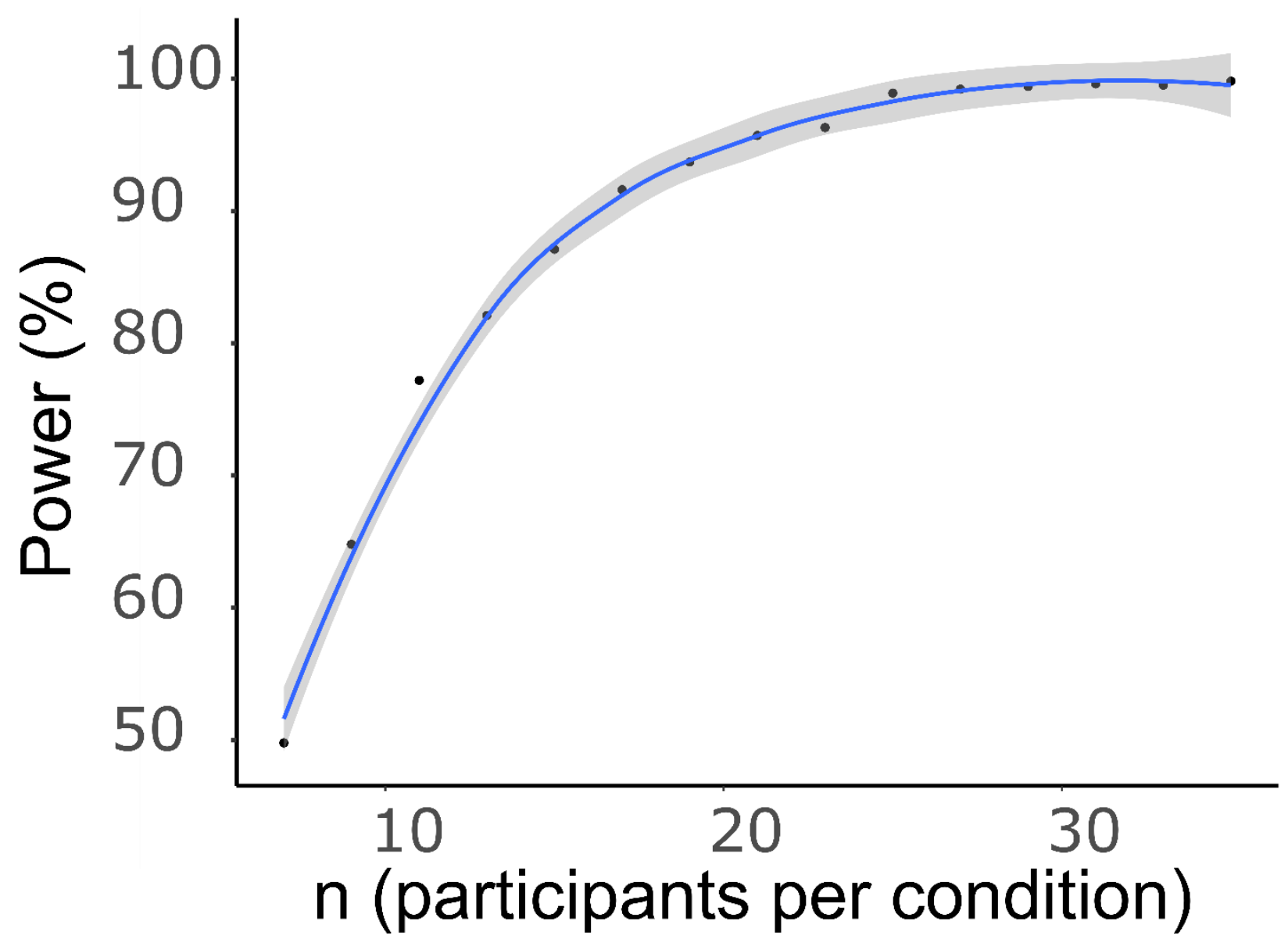

Fig. SM1. Simulation-based a-priori power estimations for different $\mathrm{n}$ participants per condition. This simulation was conducted on a conservative scenario in which the control group was equally affected by Anger Superiority, regardless of hypnotizability.

And for the hypothesis-driven simulation scenario, the power was naturally higher (as we were testing for the triple interaction, and the simulated data in this scenario considered an incremental difference in accuracy depending on hypnotizability and hypnosis). 


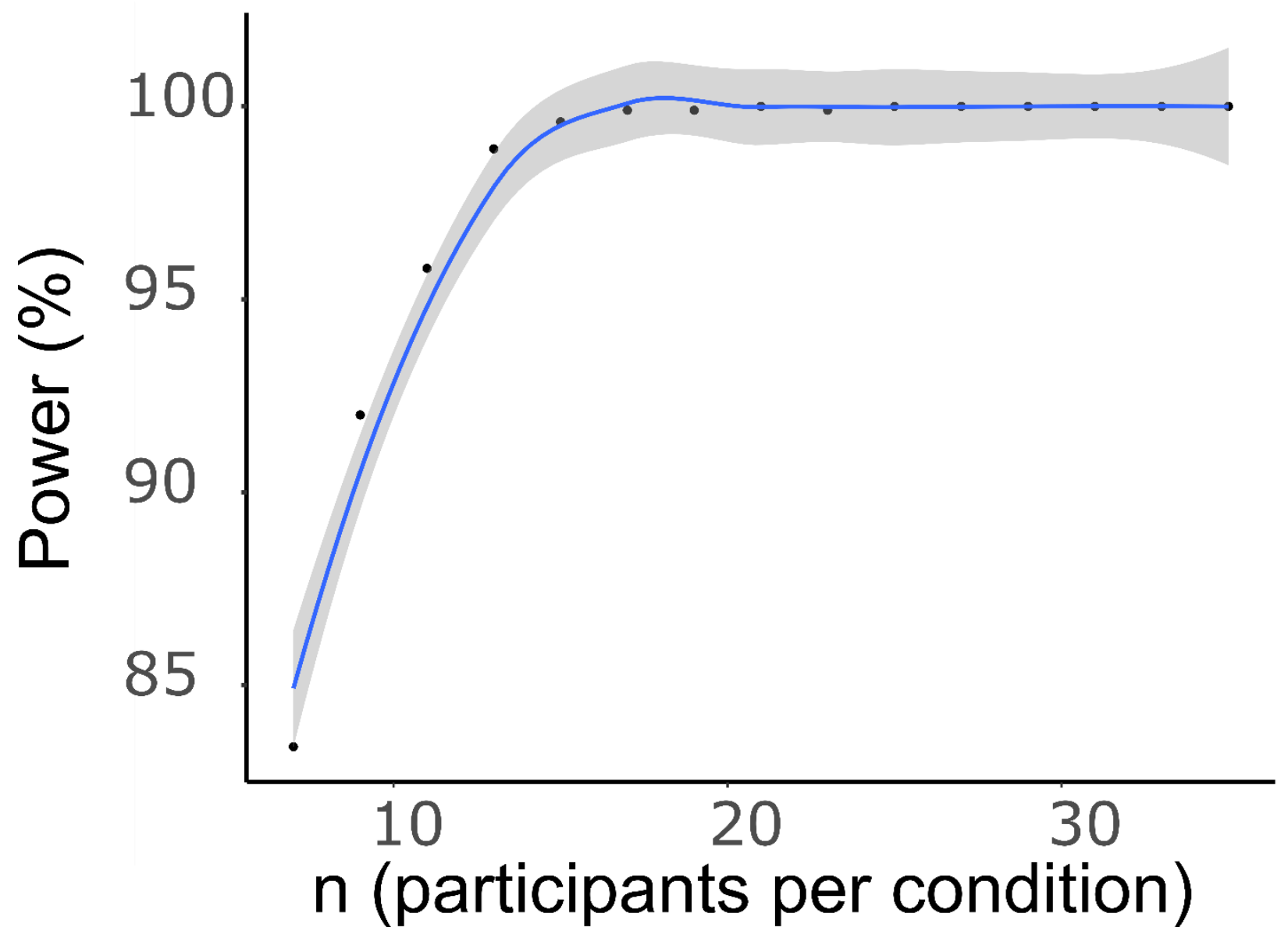

Fig. SM2. Simulation-based a-priori power estimations for different $\mathrm{n}$ participants per condition. This simulation was conducted on a hypothesis-driven scenario in which the control group was affected by Anger Superiority as a function of their hypnotizability, assuming Highs would be more permeable to AS despite its task-irrelevance.

Because $\mathrm{n}$ per condition was asymmetric in the actual data collected experimentally, we decided to replicate our experimental findings synthetically in order to obtain an approximation of observed power as an additional measure. We produced 1000 cohorts of synthetic data a total of 1000 times, for obtaining power and its $95 \% \mathrm{Cl}$. Each synthetic cohort consisted of 6 groups of participants (High, Medium and Low susceptibility, with and without hypnosis). The $\mathrm{n}$ of each group was the same as the $\mathrm{n}$ for the originally collected data (e. g., synthetic Hypnotized Lows where $n=16$ ). For each participant, we produced mean accuracy values (\% correct) for Angry and Neutral T2 trials. These datapoints were produced by 
generating n-length vectors of normally distributed random numbers, where the mean and standard deviation of the distribution where the actual mean accuracies and standard deviations observed in the original data for that group (i. e. all 1000 groups of Hypnotized Lows were different from each other, but they all had the same Angry and Neutral T2 accuracy means and SDs than the Hypnotized Lows from the real data). We modelled each synthetic group using the same model as the original data, and checked if the interaction Hypnotizability x Hypnotized x T2 was significant. Here, power was equivalent to the percentage of iterations where the interaction was significant given an alpha of 0.05 . Then, to obtain the $95 \% \mathrm{Cl}$ for the resulting power, we repeated this entire process 1000 times (yielding a total of 1000000 iterations). Our results indicated that we had a power of $91 \%(95 \% \mathrm{Cl}=90,93)$ to detect a significant Hypnosis x Hypnotizability x T2 interaction using a model identical to the one used for modelling our actual experimental data.

\subsubsection{Effect sizes}

Effect sizes were estimated by calculating Cohen's $d$ at each contrast, correcting for sample size $n<20$, so that $(1) d=\left\{\bar{x}_{1}-\bar{x}_{2} / \sqrt{ }\left[\left(s_{1}{ }^{2}+s_{2}{ }^{2}\right) / 2\right]\right\} *[(n-3) /(n-2.25)] *\{\sqrt{ }[(n-2) / n]\}$ (Cohen, 1988; Durlak, 2009). Following this computation, a $d=0.2$ is interpretable as a small effect, $d=0.5$ as a medium effect and $d=0.8$ as a large effect.

\section{Supplementary Tables}

\section{Table SM1}

Table SM1 


\begin{tabular}{|c|c|c|c|c|c|c|}
\hline Model: & \multicolumn{3}{|c|}{ Accuracy (\% correct) } & \multicolumn{3}{|c|}{ Response Times (ms) } \\
\hline$T 2_{\text {emotion }} X$ Hypnotizability $X$ & $\chi^{2}$ & DF & $\operatorname{Pr}\left(>x^{2}\right)$ & $\chi^{2}$ & DF & $\operatorname{Pr}\left(>x^{2}\right)$ \\
\hline Lag Categorv & 763 & 1 & $<0.0001$ & 196 & 1 & $<0.0001$ \\
\hline T1 $1_{\text {emotion }}$ & 27 & 1 & $<0.0001$ & 0.7 & 1 & 0.4 \\
\hline T2 emotion & 70 & 1 & $<0.0001$ & 8 & 1 & $<0.01$ \\
\hline Hypnotizability & 2 & 2 & 0.3 & 4 & 2 & 0.1 \\
\hline Hypnotized & 2 & 1 & 0.2 & 1 & 1 & 0.3 \\
\hline Lag Category x T1 $1_{\text {emotion }}$ & 2 & 1 & 0.2 & 0.2 & 1 & 0.7 \\
\hline Lag Category x T2 emotion & 0 & 1 & 1 & 0.6 & 1 & 0.4 \\
\hline T1 $1_{\text {emotion }} \times \mathrm{T} 2$ emotion & 7 & 1 & $<0.05$ & 0 & 1 & 1 \\
\hline Lag Category $x$ & 9 & 2 & $<0.01$ & 0 & 1 & 1 \\
\hline T1 emotion x Hypnotizability & 3 & 2 & 0.2 & 0.8 & 2 & 0.7 \\
\hline T2 emotion x Hypnotizability & 1 & 2 & 0.5 & 2 & 2 & 0.4 \\
\hline Lag Category x Hypnotized & 2 & 1 & 1.7 & 17 & 1 & $<0.0001$ \\
\hline T1 emotion $x$ Hypnotized & 0 & 1 & 1 & 1 & 1 & 0.4 \\
\hline T2emotion $x$ Hypnotized & 5 & 1 & $<0.05$ & 9 & 1 & $<0.01$ \\
\hline Hypnotizability $x$ & 1 & 2 & 0.5 & 7 & 2 & $<0.05$ \\
\hline Lag C x T1 $1_{\text {emotion }} \times \mathrm{T} 2$ emotion & 0.2 & 2 & 0.7 & 1 & 1 & 0.3 \\
\hline $\begin{array}{l}\text { Lag C x T1 } 1_{\text {emotion }} \times \\
\text { Hypnotizability }\end{array}$ & 1.6 & 2 & 0.4 & 2 & 2 & 0.4 \\
\hline $\begin{array}{l}\text { Lag C x T2 emotion X } \\
\text { Hypnotizability }\end{array}$ & 0.3 & 2 & 0.8 & 2 & 2 & 0.4 \\
\hline $\begin{array}{l}T 1_{\text {emotion }} \times T 2_{\text {emotion }} X \\
\text { Hypnotizability }\end{array}$ & 0.1 & 2 & 0.9 & 2 & 2 & 0.4 \\
\hline $\begin{array}{l}\text { Lag C x T1 } 1_{\text {emotion }} \times \\
\text { Hypnotized }\end{array}$ & 0.2 & 1 & 0.7 & 0.1 & 1 & 0.8 \\
\hline $\begin{array}{l}\text { Lag C x T2 emotion X } \\
\text { Hypnotized }\end{array}$ & 0.2 & 1 & 0.7 & 1 & 1 & 0.3 \\
\hline
\end{tabular}




\begin{tabular}{|c|c|c|c|c|c|c|}
\hline $\begin{array}{l}\mathrm{T} 1_{\text {emotion }} \times \mathrm{T} 2_{\text {emotion }} \mathrm{X} \\
\text { Hypnotized }\end{array}$ & 0.3 & 1 & 0.6 & 0.3 & 1 & 0.6 \\
\hline $\begin{array}{l}\text { Lag C x Hyptzd x } \\
\text { Hypnotizability }\end{array}$ & 8 & 2 & $<0.05$ & 0.1 & 2 & 1 \\
\hline $\begin{array}{l}\text { T1 emotion } x \text { Hyptzd x } \\
\text { Hypnotizability }\end{array}$ & 4 & 2 & 0.15 & 2 & 2 & 3 \\
\hline $\begin{array}{l}\text { T2 emotion } x \text { Hyptzd } x \\
\text { Hypnotizability }\end{array}$ & 14 & 2 & $<0.01$ & 5.5 & 2 & 0.07 \\
\hline $\begin{array}{l}\text { Lag C x T1 } 1_{\text {emotion }} \times \mathrm{T} 2_{\text {emotion }} \times \\
\text { Hyptzblty }\end{array}$ & 0.7 & 2 & 0.7 & 0.9 & 2 & 0.6 \\
\hline $\begin{array}{l}\text { Lag C } \times \mathrm{T} 1_{\text {emotion }} \times \mathrm{T} 22_{\text {emotion }} \times \\
\text { Hyptzd }\end{array}$ & 0.2 & 1 & 0.6 & 3.2 & 1 & 0.07 \\
\hline $\begin{array}{l}\text { Lag C } \times \text { T1 } 1_{\text {emotion }} \times \text { Hyptzblty } \\
\text { x Hyptzd }\end{array}$ & 0.2 & 2 & 0.9 & 5 & 2 & 0.08 \\
\hline $\begin{array}{l}\text { Lag C } \times \text { T2 } 2_{\text {emotion }} \times \text { Hyptzblty } \\
\text { x Hyptzd }\end{array}$ & 7 & 2 & $<0.05$ & 3 & 2 & 0.2 \\
\hline $\begin{array}{l}\mathrm{T} 1_{\text {emotion }} \times \mathrm{T} 2_{\text {emotion }} \mathrm{x} \\
\text { Hyptzblty } \times \text { Hyptzd }\end{array}$ & 0.4 & 2 & 0.8 & 1 & 2 & 0.5 \\
\hline Lag C x T1 $1_{\text {emotion }} \times \mathrm{T} 2_{\text {emotion }} \times$ & & & & & & \\
\hline Hyptzblty x Hyptzd & 2 & 2 & 0.3 & 0.7 & 2 & 0.7 \\
\hline
\end{tabular}

Table SM 1. Full array of effects and interactions over accuracy and response times for the main task (inclination orientation), according to the model "Accuracy / Response Times Lag Category $\mathrm{X} T 1_{\text {emotion }} \mathrm{X}$ Hypnotizability $\mathrm{X}$ Hypnotized $+\varepsilon^{\prime \prime}$. ANOVA tables were computed through Analysis of Deviance (Type II Wald $\chi^{2}$ test). In pink, all statistically significant effects and interactions.

\section{Table SM2}

Accuracy (\%) Lag Category X T1 $1_{\text {emotion }}$ X T2 emotion X Hypnotizability X Hypnotized $+\varepsilon$ 


\begin{tabular}{|c|c|c|c|}
\hline & $x^{2}$ & DF & $\operatorname{Pr}\left(>x^{2}\right)$ \\
\hline Lag Category & 3850 & 1 & $<0.0001$ \\
\hline $\mathrm{T} 1_{\text {emotion }}$ & 111 & 1 & $<0.0001$ \\
\hline T2 $2_{\text {emotion }}$ & 216 & 1 & $<0.0001$ \\
\hline Hypnotizability & 2.5 & 2 & 0.3 \\
\hline Hypnotized & 3.7 & 1 & 0.07 \\
\hline Lag Category x T1 $1_{\text {emotion }}$ & 45 & 1 & $<0.0001$ \\
\hline Lag Category x T2 emotion & 18 & 1 & $<0.0001$ \\
\hline $\mathrm{T} \mathbf{1}_{\text {emotion }} \times \mathrm{T} \mathbf{2}_{\text {emotion }}$ & 56 & 1 & $<0.0001$ \\
\hline Lag Category x Hypnotizability & 2 & 2 & 0.3 \\
\hline T1 emotion $x$ Hypnotizability & 1 & 2 & 0.6 \\
\hline T2 emotion $x$ Hypnotizability & 8 & 2 & $<0.05$ \\
\hline Lag Category x Hypnotized & 5.5 & 1 & $<0.05$ \\
\hline T1 emotion $x$ Hypnotized & 4 & 1 & $<0.05$ \\
\hline T2 emotion $x$ Hypnotized & 0.8 & 1 & 0.4 \\
\hline Hypnotizability x Hypnotized & 3 & 2 & 0.2 \\
\hline Lag C x T1 $1_{\text {emotion }} \times \mathrm{T} 2$ emotion & 0.2 & 1 & 0.7 \\
\hline Lag C x T1 emotion $\times$ Hypnotizability & 4 & 2 & 0.1 \\
\hline Lag C x T2 emotion $x$ Hypnotizability & 1 & 2 & 0.5 \\
\hline T1 $1_{\text {emotion }} \times$ T2 emotion Hypnotizability & 4 & 2 & 0.1 \\
\hline Lag C x T1 emotion $\times$ Hypnotized & 1.7 & 1 & 0.2 \\
\hline Lag C x T2 emotion $x$ Hypnotized & 2 & 1 & 0.1 \\
\hline $\mathrm{T} 1_{\text {emotion }} \times \mathrm{T} 2_{\text {emotion }} \times$ Hypnotized & 4 & 1 & $<0.05$ \\
\hline Lag C x Hyptzd x Hypnotizability & 5 & 2 & 0.07 \\
\hline T1 $1_{\text {emotion }} \times$ Hyptzd x Hypnotizability & 5 & 2 & 0.07 \\
\hline T2 emotion x Hyptzd x Hypnotizability & 4 & 2 & 0.16 \\
\hline Lag C x T1 $1_{\text {emotion }} \times$ T2 emotion $x$ Hyptzblty & 1 & 2 & 0.5 \\
\hline Lag C $\times$ T1 $1_{\text {emotion }} \times \mathrm{T} 2_{\text {emotion }} \times$ Hyptzd & 0.04 & 1 & 0.8 \\
\hline Lag C x T1 emotion $\times$ Hyptzblty x Hyptzd & 0.4 & 2 & 0.8 \\
\hline Lag C x T2 emotion $x$ Hyptzblty x Hyptzd & 0.07 & 2 & 1 \\
\hline
\end{tabular}


T1 $1_{\text {emotion }} \times$ T2 emotion $_{\text {X Hyptzblty }} \times$ Hyptzd

Lag C $\times$ T1 $1_{\text {emotion }} \times$ T2 emotion $\times$ Hyptzblty $\times$ Hyptzd
0.4

0.9

2

0.6

Table SM 2. Full array of effects and interactions over accuracy for the secondary task (target counting), according to the model "Accuracy Lag Category $X \mathrm{~T} 1_{\text {emotion }} \times$ Hypnotizability $X$ Hypnotized $+\varepsilon^{\prime \prime}$. ANOVA tables were computed through Analysis of Deviance (Type II Wald $\chi^{2}$ test). In pink, all statistically significant effects and interactions.

\section{Table SM3}

$\sim$ Lag Category X T1 $1_{\text {emotion }}$ X T2 $2_{\text {emotion }}$ X Hypnotizability X Hypnotized $+\varepsilon$

\begin{tabular}{|c|c|c|c|c|c|c|c|c|c|}
\hline & $\underline{a}$ & & & $\underline{\boldsymbol{v}}$ & & & $\underline{t 0}$ & & \\
\hline & $x^{2}$ & DF & $\operatorname{Pr}\left(>x^{2}\right)$ & $x^{2}$ & DF & $\operatorname{Pr}\left(>x^{2}\right)$ & $x^{2}$ & DF & $\operatorname{Pr}\left(>x^{2}\right)$ \\
\hline Lag Category & 192 & 1 & $<0.0001$ & 423 & 1 & $<0.0001$ & 64 & 1 & $<0.0001$ \\
\hline T1 1 emotion & 0.17 & 1 & 0.7 & 12 & 1 & $<0.001$ & 1.7 & 1 & 0.2 \\
\hline T2emotion & 5 & 1 & $<0.05$ & 34 & 1 & $<0.0001$ & 4.7 & 1 & $<0.05$ \\
\hline Hypnotizability & 5 & 2 & 0.08 & 1.6 & 2 & 0.5 & 1.5 & 2 & 0.5 \\
\hline Hypnotized & 4 & 1 & $<0.05$ & 5 & 1 & $<0.05$ & 4.7 & 1 & $<0.05$ \\
\hline
\end{tabular}




\begin{tabular}{|c|c|c|c|c|c|c|c|c|c|}
\hline $\begin{array}{l}\text { Lag Category } x \\
T 1_{\text {emotion }}\end{array}$ & 0.35 & 1 & 0.6 & 2 & 1 & 0.12 & 0.5 & 1 & 0.5 \\
\hline $\begin{array}{l}\text { Lag Category x } \\
\text { T2emotion }\end{array}$ & 5 & 1 & $<0.05$ & 2 & 1 & 0.18 & 1.6 & 1 & 0.2 \\
\hline $\mathrm{T} 1_{\text {emotion }} \times \mathrm{T} \mathbf{2}_{\text {emotion }}$ & 0.4 & 1 & 0.5 & 4.6 & 1 & $<0.05$ & 0.2 & 1 & 0.6 \\
\hline $\begin{array}{l}\text { Lag Category x } \\
\text { Hypnotizability }\end{array}$ & 2 & 2 & 0.4 & 6 & 2 & $=0.05$ & 0.2 & 2 & 0.9 \\
\hline $\begin{array}{l}\text { T1emotion } x \\
\text { Hypnotizability }\end{array}$ & 2 & 2 & 0.3 & 0.06 & 2 & 0.97 & 2.8 & 2 & 0.2 \\
\hline $\begin{array}{l}\text { T2 emotion X } \\
\text { Hypnotizability }\end{array}$ & 0.5 & 2 & 0.8 & 0.7 & 2 & 0.71 & 0.6 & 2 & 0.7 \\
\hline $\begin{array}{l}\text { Lag Category x } \\
\text { Hypnotized }\end{array}$ & 7 & 1 & $<0.01$ & 5 & 1 & $<0.05$ & 0.1 & 1 & 0.7 \\
\hline $\begin{array}{l}\text { T1 emotion X } \\
\text { Hypnotized }\end{array}$ & 3 & 1 & 0.09 & 0.04 & 1 & 0.84 & 1.7 & 1 & 0.2 \\
\hline $\begin{array}{l}\text { T2emotion X } \\
\text { Hypnotized }\end{array}$ & 0.45 & 1 & 0.5 & 1 & 1 & 0.25 & 0.3 & 1 & 0.5 \\
\hline $\begin{array}{l}\text { Hypnotizability x } \\
\text { Hypnotized }\end{array}$ & 6 & 2 & $<0.05$ & 4 & 2 & 0.11 & 0.8 & 2 & 0.7 \\
\hline $\begin{array}{l}\text { Lag C x T1 emotion } x \\
\text { T2 emotion }\end{array}$ & 0.5 & 1 & 0.5 & 0.2 & 1 & 0.7 & 3 & 1 & 0.08 \\
\hline $\begin{array}{l}\text { Lag C x T1 } 1 \text { emotion } x \\
\text { Hypnotizability }\end{array}$ & 0.3 & 2 & 0.9 & 1.7 & 2 & 0.4 & 0 & 2 & 1 \\
\hline $\begin{array}{l}\text { Lag C x T2 emotion X } \\
\text { Hypnotizability }\end{array}$ & 0.3 & 2 & 0.8 & 0.4 & 2 & 0.8 & 1.2 & 2 & 0.6 \\
\hline
\end{tabular}




\begin{tabular}{|c|c|c|c|c|c|c|c|c|c|}
\hline $\begin{array}{l}\mathrm{T} 1_{\text {emotion }} \text { X T2 } \text { emotion } \\
\text { Hypnotizability }\end{array}$ & 3 & 2 & 0.2 & 0.1 & 2 & 0.9 & 7 & 2 & $<0.05$ \\
\hline $\begin{array}{l}\text { Lag C x T1 } 1_{\text {emotion }} \times \\
\text { Hypnotized }\end{array}$ & 1 & 1 & 0.3 & 0.01 & 1 & 0.9 & 0.4 & 1 & 0.5 \\
\hline $\begin{array}{l}\text { Lag C x T2 emotion X } \\
\text { Hypnotized }\end{array}$ & 0.4 & 1 & 0.5 & 0.4 & 1 & 0.6 & 0.02 & 1 & 0.9 \\
\hline $\begin{array}{l}T 1_{\text {emotion }} \times T 2_{\text {emotion }} \times \\
\text { Hypnotized }\end{array}$ & 0.5 & 1 & 0.5 & 0.4 & 1 & 0.6 & 0.2 & 1 & 0.7 \\
\hline $\begin{array}{l}\text { Lag C x Hyptzd x } \\
\text { Hypnotizability }\end{array}$ & 1.6 & 2 & 0.5 & 3 & 2 & 0.2 & 15 & 2 & $<0.001$ \\
\hline $\begin{array}{l}\text { T1 emotion } x \text { Hyptzd x } \\
\text { Hypnotizability }\end{array}$ & 2 & 2 & 0.3 & 4 & 2 & 0.14 & 0.9 & 2 & 0.6 \\
\hline $\begin{array}{l}\text { T2 emotion } x \text { Hyptzd x } \\
\text { Hypnotizability }\end{array}$ & 0.01 & 2 & 1 & 4 & 2 & 0.14 & 1 & 2 & 0.6 \\
\hline $\begin{array}{l}\text { Lag C x T1 } 1_{\text {emotion }} \times \\
\text { T2 } \text { emotion } \text {. Hyptzblty }\end{array}$ & 0.2 & 2 & 0.9 & 1 & 2 & 0.5 & 0.8 & 2 & 0.7 \\
\hline $\begin{array}{l}\text { Lag C x T1 } 1_{\text {emotion }} \times \\
\text { T2 } \\
\text { emotion } \times \text { Hyptzd }\end{array}$ & 0.01 & 1 & 0.9 & 0.6 & 1 & 0.5 & 0.8 & 1 & 0.4 \\
\hline $\begin{array}{l}\text { Lag C x T1 } 1_{\text {emotion }} \times \\
\text { Hyptzblty x Hyptzd }\end{array}$ & 2 & 2 & 0.4 & 1.6 & 2 & 0.5 & 3.7 & 2 & 0.2 \\
\hline $\begin{array}{l}\text { Lag C x T2 emotion X } \\
\text { Hyptzblty x Hyptzd }\end{array}$ & 3.6 & 2 & 0.16 & 1.7 & 2 & 0.4 & 0.4 & 2 & 0.8 \\
\hline $\begin{array}{l}\mathrm{T} 1_{\text {emotion }} \times \mathrm{T} 2_{\text {emotion }} \times \\
\text { Hyptzblty } \times \text { Hyptzd }\end{array}$ & 2 & 2 & 0.4 & 0.2 & 2 & 0.9 & 0.5 & 2 & 0.8 \\
\hline $\begin{array}{l}\text { Lag C x T1 } 1_{\text {emotion }} \times \\
\text { T2 } \\
\text { emotion } \times \text { Hyptzblty }\end{array}$ & 2 & 2 & 0.4 & 0.7 & 2 & 0.7 & 1 & 2 & 0.6 \\
\hline
\end{tabular}


x Hyptzd

Table SM 3. Full array of effects and interactions over $a$, tO and $v$, according to the model " $a / t O / v$

$\sim$ Lag Category X T1 $1_{\text {emotion }}$ X Hypnotizability X Hypnotized $+\varepsilon "$. ANOVA tables were computed through Analysis of Deviance (Type II Wald $\chi^{2}$ test). In pink, all statistically significant effects and interactions.

\section{Additional supplementary figures}




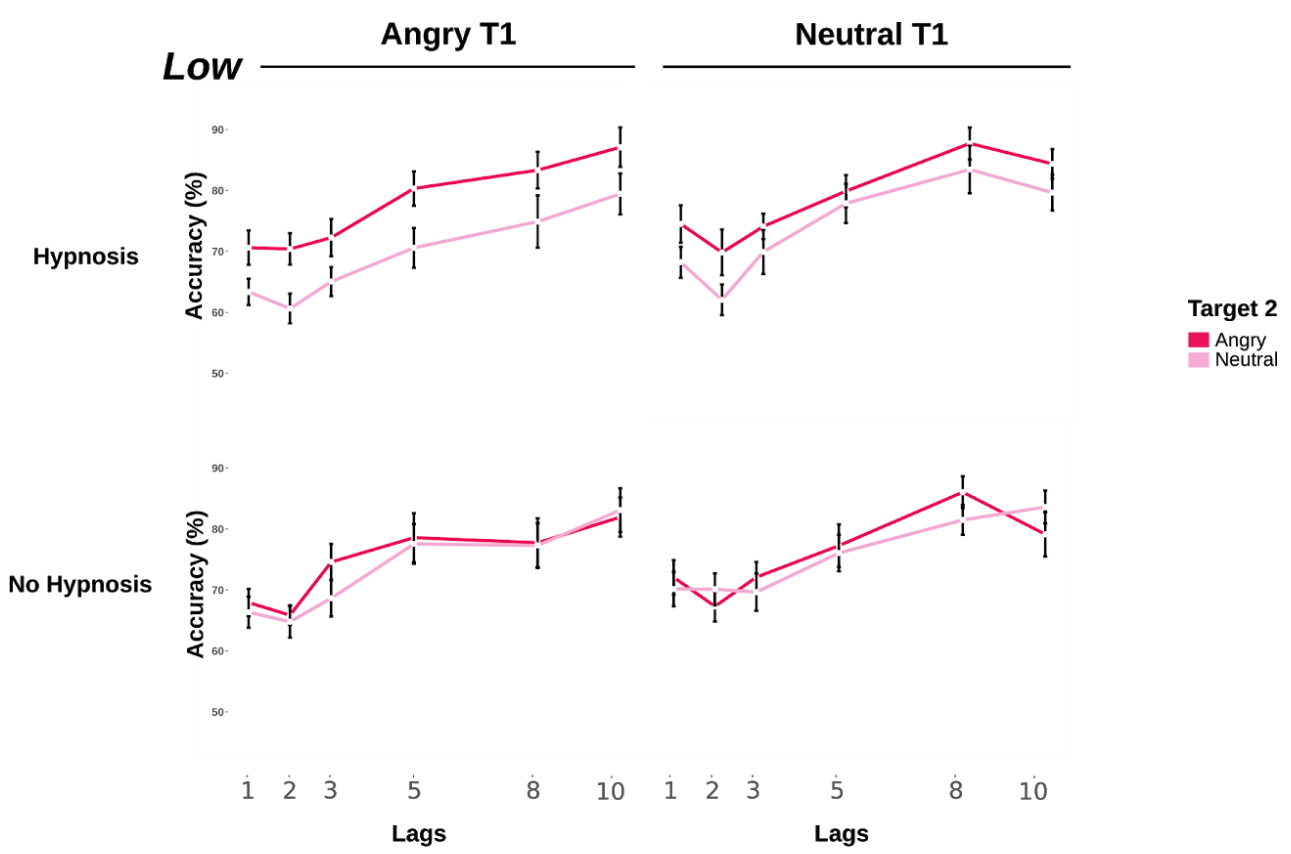

\section{Medium}

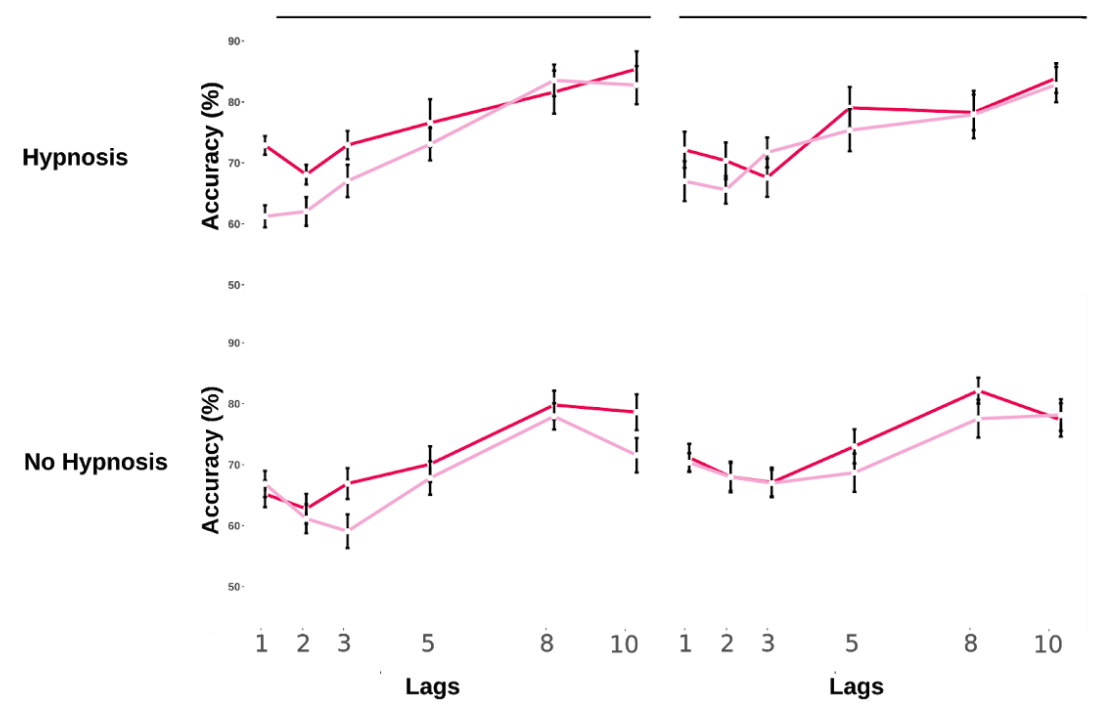

Target 2

\section{High}

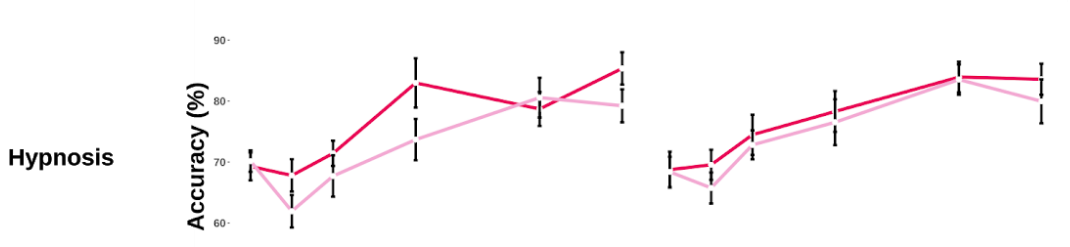

Target 2

Angry
Neutra

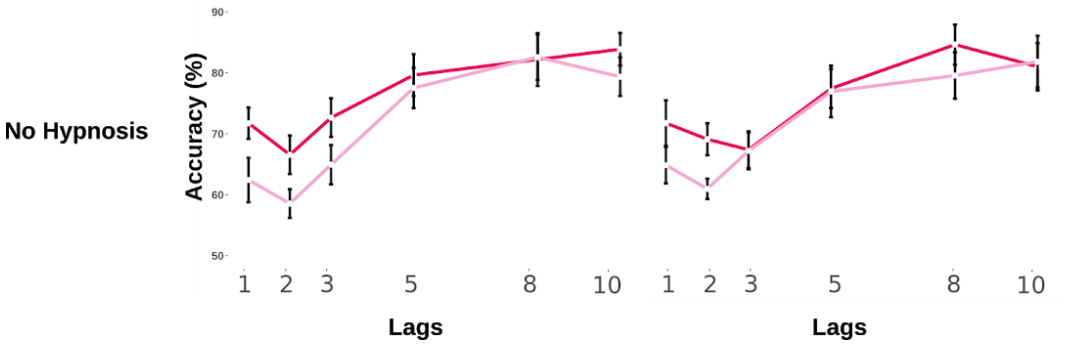




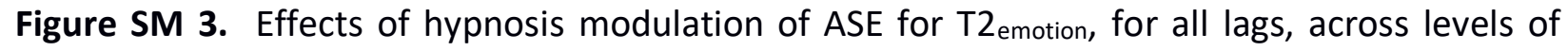
$\mathrm{T} 1_{\text {emotion. }} \mathrm{T} 1_{\text {emotion }}$ did not interact significantly with the Hypnotized and Hypnotizability factors.

\section{Hypnotized Non-Hypnotized}

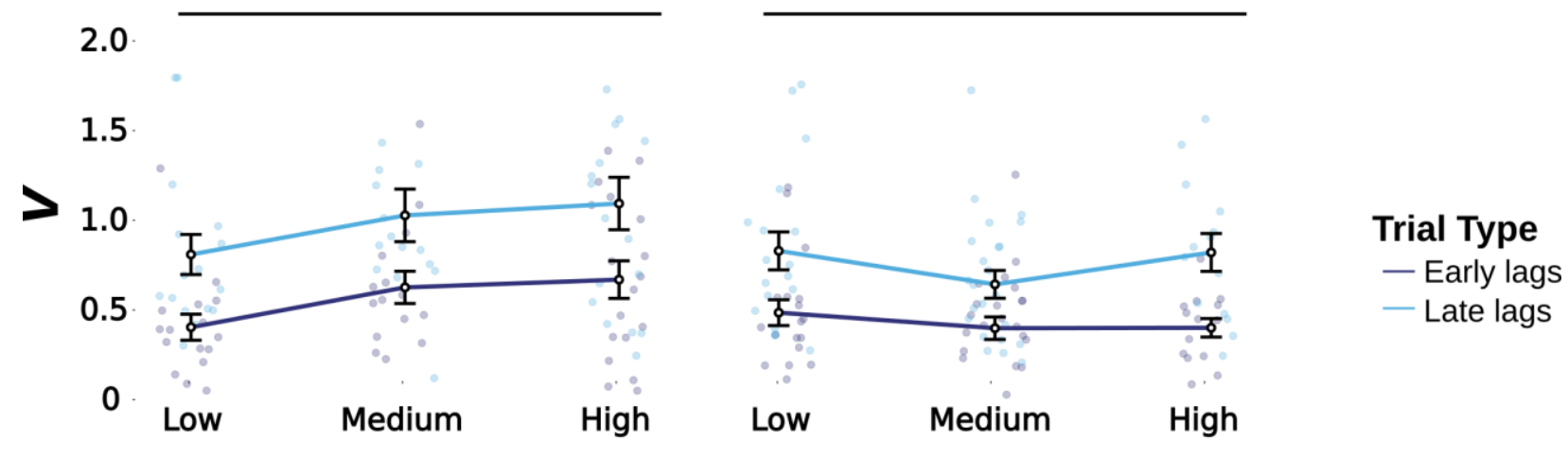

Figure SM4. $v$ plotted across Lag Category and Hypnotizability, for hypnotized and nonhypnotized participants. 


\section{References}

Agresti, A. (2002) Categorical Data Analysis. Wiley, second edition.

Anderson A. (2005) Affective Influences on the Attentional Dynamics Supporting Awareness. Journal of Experimental Psychology: General, Vol. 134, No. 2, 258-281. DOI: 10.1037/00963445.134 .2 .258

Anlló H., Becchio J., Sackur J. (2017) French norms for the Harvard Group Scale of Hypnotic Susceptibility, form A, International Journal of Clinical and Experimental Hypnosis.

Bates D., Maechler M., Bolker B., Walker S. (2015) Fitting Linear Mixed-Effects Models Using Ime4. Journal of Statistical Software, 67(1), 1-48.

Bolker B., Brooks M., Clark C., Geange S., Poulsen j., Stevens H., White J. (2008) Generalized linear mixed models: a practical guide for ecology and evolution Trends in Ecology and Evolution Vol.24 No.3

Cohen, J. (1988). Statistical power analysis for the behavioral sciences. Routledge, 2nd Ed. ISBN 0-8058-0283-5.

de Jong P., Martens S. (2007) Detection of emotional expressions in rapidly changing facial displays in high- and low-socially anxious women. Behaviour Research and Therapy 45, 12851294. doi:10.1016/j.brat.2006.10.003

Durlak, J. (2009) How to Select, Calculate, and Interpret Effect Sizes. Journal of Pediatric Psychology. March: 34(9):917-28. 
Fox J., Weisberg S. (2011). An $\{$ R $\}$ Companion to Applied Regression, Second Edition. Thousand Oaks CA: Sage. URL: http://socserv.socsci.mcmaster.ca/jfox/Books/Companion

Iani C, Ricci F. Baroni G., Rubichi S., (2009) Attention control and susceptibility to hypnosis. Consciousness and Cognition 18 (2009) 856-863. doi:10.1016/j.concog.2009.07.002

Jaeger, T. (2008). Categorical data analysis: Away from ANOVAs (transformation or not) and towards logit mixed models. Journal of Memory and Language, 59(4):434-446.

Jensen P., Jamieson G., Lutz A., Mazzoni G., McGeown W., Santarcangelo E., Demertzi A., De Pascalis V., Bányai E., Rominger C., Vuilleumier P., Faymonville M., Terhune D. (2017) New directions in hypnosis research: strategies for advancing the cognitive and clinical neuroscience of hypnosis, Neuroscience of Consciousness, Volume 2017, Issue 1, nix004, https://doi.org/10.1093/nc/nix004

Kanade, T., Cohn, J. F., Tian, Y. (2000). Comprehensive database for facial expression analysis. Proceedings of the Fourth IEEE International Conference on Automatic Face and Gesture Recognition (FG'00), Grenoble, France, 46-53.

Langner, O., Dotsch, R., Bijlstra, G., Wigboldus, D.H.J., Hawk, S.T., \& van Knippenberg, A. (2010). Presentation and validation of the Radboud Faces Database. Cognition \& Emotion, 24(8), 1377-1388. DOI: 10.1080/02699930903485076

Lenth R. (2016). Least-Squares Means: The R Package Ismeans. Journal of Statistical Software, 69(1), 1-33.

Lucey, P., Cohn, J. F., Kanade, T., Saragih, J., Ambadar, Z., \& Matthews, I. (2010). The Extended Cohn-Kanade Dataset $(\mathrm{CK}+)$ : A complete expression dataset for action unit and emotion- 
specified expression. Proceedings of the Third International Workshop on CVPR for Human Communicative Behavior Analysis (CVPR4HB 2010), San Francisco, USA, 94-101.

Ma D. S., Correll J., Wittenbrink B. (2015) The Chicago face database: A free stimulus set of faces and norming data. Behav Res Methods. Dec;47(4):1122-35. doi: 10.3758/s13428-0140532-5.

Martinez A., Benavente R. (1998) The AR Face Database. CVC Technical Report \#24, June.

Pinheiro J.C., Bates D.M. (2000). Mixed-Effects Models in S and SPLUS. New York: Springer.

R Development Core Team (2008). R: A language and environment for statistical computing. R Foundation for Statistical Computing, Vienna, Austria. ISBN 3-900051-07-0, URL http://www.R-project.org.

Ratcliff, R. (1978). A theory of memory retrieval. Psychological Review, 85, 59-108.

Shor R., Orne E. (1962) Harvard Group Scale of Hypnotic Susceptibility, form A, Consulting Psychologists Press, Inc. Palo Alto, California.

Stein T., Zwickel J., Ritter J., Kitzmantel M., Schneider W (2009) The effect of fearful faces on the attentional blink is task dependent. Psychonomic Bulletin \& Review, 16 (1), 104-109 doi:10.3758/PBR.16.1.104

Taylor J, Whalen P. (2014) Fearful, But Not Angry, Expressions Diffuse Attention to Peripheral Targets in an Attentional Blink Paradigm Emotion, Vol. 14, No. 3, 462-468. DOI: $10.1037 / a 0036034$

Tottenham N., Tanaka J. W., Leon A. C., McCarry T., Nurse M., Hare T. A., Marcus D. J., Westerlund A., Casey B. J., Nelson C. (2009) The NimStim set of facial expressions: judgments 
from untrained research participants. Psychiatry Res. Aug 15;168(3):242-9. doi: 10.1016/j.psychres.2008.05.006.

van der Schalk J., Hawk S. T., Fischer A. H., Doosje B. (2011), Moving Faces, Looking Places: Validation of the Amsterdam Dynamic Facial Expression Set (ADFES), Emotion (C) 2011 American Psychological Association, Vol. 11, No. 4, 907-920.

Voss, A., Rothermund, K., \& Voss, J. (2004) Interpreting the parameters of the diffusion model: An empirical validation. Memory and Cognition, 32, 1206-1220.

Willenbockel V., Sadr J., Fiset D., Horne G. O., Gosselin F., Tanaka J. W. (2010) Controlling lowlevel image properties: the SHINE toolbox. Behav Res Methods. Aug;42(3):671-84. doi: 10.3758/BRM.42.3.671.

Yao S., Ding C., Qi S., Yang D (2014) Value associations of emotional faces can modify the anger superiority effect: behavioral and electrophysiological evidence. SCAN (2014) 9, 849-856. doi:10.1093/scan/nst056 
Appendix B - Hypnotic induction and suggestion

$\underline{1^{\text {st }} \text { Induction (based on the Elman induction) }}$

Stage 1 
The participant is asked to sit comfortably and relax. She's then asked to slow her breathing and consciously take control of it. She's asked to make it deep, regular and refreshing. The operator, sitting by her side, then tells her that during the procedure that he will be engaging on some forms of mild physical contact and demonstrates

"Donc, Il se trouve que pendant l'activation de la procédure hypnotique je vais peutêtre vous toucher gentiment ici [touches wrist], ici [touches shoulder] et ici [touches forehead] avec votre permission. C'est très bien. " [So then, it so just happens that during the launching of the hypnotic procedure I may touch you here [touches wrist], here [touches shoulder] and here [touches forehead], with your permission of course. That's perfect.]

The operator brings his right hand close to the participant's face, vertically, keeping it at approximately $10 \mathrm{~cm}$ from her forehead, and asks her to fixate her gaze on the top of his middle finger. It is crucial that the participant forces herself to look up in order to be able to maintain visual contact with the aforementioned target. The participant is then told to concentrate as much as possible on the operator's fingertip, and she's instructed to breathe increasingly deeper and slower. She's then told to get ready to experience a "descending wave" of relaxation from the head to the tip of her toes, which will come the moment she closes her eyes. The operator then takes a brief moment to describe this pleasant sensation, in order to give the participant's eyes enough time to get tired and strained.

\section{Stage 2}

The operator gets ready to enact the interactive relaxation procedure 
"Maintenant, je vais rapprocher ma main de vos yeux de façon descendante. II serait bien si vous permettez à votre corps de se relaxer en fermant vos yeux en même temps. Les fermer en suivant le mouvement de ma main. Très bien. [approaches hand towards eyes in a descending fashion, almost like closing them, but without touching them] Vous allez ressentir sans doute comment ils se ferment, et la détente de vos muscles oculaires... elle se propage d'elle-même vers tous les muscles de votre corps. Très bien. Comme une vague qui descende jusqu'au bout de vos orteils. Ressentez cette sensation très agréable. " [Now, I shall bring my hand close to your eyes in a descending fashion. It would be great if you'd allow your body to be completely relaxed as you close your eyes... As you close them following the movement of my hand. That's great [approaches hand towards eyes in a descending fashion, almost like closing them, but without touching them]. You will certainly feel how they close, and your eyes' muscles relaxation... propagates all the way down to the tip of your toes. Indulge in this pleasant sensation... ]

Now, the operator will hold his hand horizontally, over the participant's closed eyes. The operator should try to block her potential visual field as much as possible. The operator will then explain that even though the participant's eyes feel quite heavy and tight-shut, he will be asking her to make the effort of opening them. Even if it may come as a very hard thing to do. Once the participant manages to open her eyes, she'll see that the operator's hand is blocking her view. The operator should not make any mention of this fact. She will simply congratulate the participant on being able to open her eyes, and with a descending fashion of his palm he will tell her to close them again, following the movement. The operator will then inform that with each closing motion, the hypnotic process becomes more solid, and so does the relaxation. This will be done 6 (six) times. 


\section{Stage 3}

Then, the operator will grab the participant gently by the wrist and lift her arm. The arm should feel like a limp rag. The operator will then tell the participant that he's going to let the arm fall, and that it is very possible that she may feel like her arm is somewhat independent form her body. Once the arm falls, the participant will be told that the activation of the trance shall increase five-fold. This will be done once per arm.

\section{Stage 4}

The participant is then asked to try to count backwards from a hundred to one in a very precise way

"Très bien. Maintenant, pour installer la trance active de manière complète et totale, vous allez compter à rebours depuis cent. Mais vous allez le faire en suivant mes instructions. Vous allez essayer de prononcer chaque chiffre de manière douce mais audible, et vous allez vous en servir vous-même de ce compte pour faire le travail d'activer le processus. Écoutez-moi bien, je veux que vous poussez les chiffres dehors votre esprit, dehors votre conscience. Pour chaque chiffre que vous allez mentionner, vous allez avancer plus ver cette état, et l'expansion du processus va pousser les chiffres dehors votre esprit. Peut-être que vous allez visualiser comment elles s'évanouirent, ou vous allez les oublier tout de suite... Mais dans tous les cas vous finirez par devenir incapable de continuer le compte à rebours, car les chiffres ne seront plus là, tout simplement. Et ça c'est très bien. Commencez, s'il vous plaît." [Excellent. Now, in order 
to install this state in a complete and absolute fashion, you will count backwards, starting at one hundred. But you will do it following my instructions. You will try to pronounce each number ever so gently but yet audibly, and you will use this count to work towards the consolidation of the process. Listen to me very carefully please, I want you to push each number you pronounce outside your mind, outside your consciousness. For every number you will mention, you will advance further into this state, and the expansion of the state itself will push the numbers outside of your mind. Maybe you'll see them disappearing, or you'll just forget them immediately... But no matter what you'll end up becoming incapable of finishing the count, because the numbers will no longer be there. And that's fine. Start, please.]

At every step, whenever the participant manages to count, the operator will insist gently on the increasing relaxation and departure of the numbers. Soon enough, the participant should become unable to count. At this point we consider that the induction is complete. If for whatever reason, a sensitive participant manages to count beyond 80 (which is quite rare) then the operator can choose between going back to Stage 3 or excluding the participant.

\section{Main Suggestion}

This main suggestion is oriented towards the goal of the Attentional Blink experiment.

"Maintenant vous allez ressentir plusieurs choses que je vais vous décrire. Vous allez ressentir comment avec cet état d'hypnose que vous avez réussi sans doute à installer et vérifier à travers cette espèce d'amnésie que vous venez de mettre en place, une grande 
sensation de sécurité s'installe dans votre tête, dans votre corps, dans votre esprit Cette sécurité, cette protection, elle est totale. Prenez s'il vous plaît un petit moment pour vraiment l'apprécier, la ressentir. Et pendant que vous serez dans la cabine de tests cette agréable sensation de sécurité sera avec vous de manière permanente. C'est presque comme si vous regardiez tout ce qui se passe sur l'écran et dans la salle comme derrière un verre protecteur, complètement détaché, au secours, comme depuis très très loin. Derrière une barrière de protection... Profitez vraiment de cette sécurité totale qui va vous aider à vous concentrer sur la tâche, qui va vous aider à regarder toutes les images toutes les visages en sécurité, détaché, concentrée sur la tâche. Tout ici est également inoffensif, en arrière-plan, et ça c'est très bien car comme ça vous pouvez vous détacher de tout et juste faire la tâche et ne pas vous concentrer sur autres aspects de cette cabine, de ces images. Bientôt je vais partir et dès que vous serez prête vous pourrez ouvrir vos yeux et commencer l'expérience, toujours en profitant de cette sécurité très agréable et de cet état. Dans les pauses je vais revenir vous voir pour vous aider à vous reposer et vous relaxer... et c'est très possible, même presque inévitable, que le fait d'entendre encore ma voix vous aide aussi à vous relaxer et récupérer si besoin ou encore ancrer cet état de sécurité totale et ses effets. [Now you will feel several things that I will describe to you. You will now feel how this state of hypnosis that you have certainly managed to develop and verify through this sort of amnesia that you have just enacted, comes along with a strong sense of security, strongly present in your head, in your body, in your mind. This security, this protection, it is absolute. Take if you may a moment to really appreciate how palpable this feeling is. While you'll be in this booth, you'll feel this very pleasant feeling of safety, permanently. It's as if you looked at everything that happened in here through a protective glass... like while being completely detached, in total safety, as if you looked from afar. Behind a protective barrier... Take your time to enjoy of this absolute safety that will help 
you concentrate on the task, that will help you look at every image at every face in perfect safety, detached, in perfectly concentrated on the task. Everything here is equally inoffensive, almost like background noise, and that's awesome because it will help you detach from everything and just do the task, without concentrating in any other aspect of this booth, or this images. Soon I shall leave, and as soon as you are ready you can open your eyes and start with the experiment, always while enjoying of this nice security and of this state. During the pauses I shall come back to see you and help you relax... and it is quite possible, almost inevitable, that the sound of my voice may also help you relax and recover if needed or maybe even further anchor this absolute safety and its effects.]

\section{Suggestion Reinforcement}

At every pause, the experimenter reinforced the suggestion as follows:

Très bien. Vous avez très bien travaillé jusqu'à présent. Vous allez ressentir comment avec cet état d'hypnose que vous avez réussi sans doute à installer et vérifier à travers cette espèce d'amnésie que vous venez de mettre en place, une grande sensation de sécurité s'installe dans votre tête, dans votre corps, dans votre esprit Cette sécurité, cette protection, elle est totale. Prenez s'il vous plaît un petit moment pour vraiment l'apprécier, la ressentir. Et pendant que vous serez dans la cabine de tests cette agréable sensation de sécurité sera avec vous de manière permanente. C'est presque comme si vous regardiez tout ce qui se passe sur l'écran et dans la salle comme derrière un verre protecteur, complètement détaché, au secours, comme depuis très très loin. Derrière une barrière de protection... Profitez vraiment de cette sécurité totale qui va vous aider à vous concentrer sur la tâche, qui va vous aider à regarder toutes les images toutes les visages en sécurité, détaché, concentrée sur la tâche. . [Great, you have worked splendidly so far. You will now feel how this state of hypnosis that you have 
certainly managed to develop and verify through this sort of amnesia that you have just enacted, comes along with a strong sense of security, strongly present in your head, in your body, in your mind. This security, this protection, it is absolute. Take if you may a moment to really appreciate how palpable this feeling is. While you'll be in this booth, you'll feel this very pleasant feeling of safety, permanently. It's as if you looked at everything that happened in here through a protective glass... like while being completely detached, in total safety, as if you looked from afar. Behind a protective barrier... Take your time to enjoy of this absolute safety that will help you concentrate on the task, that will help you look at every image at every face in perfect safety, detached, in perfectly concentrated on the task.] 